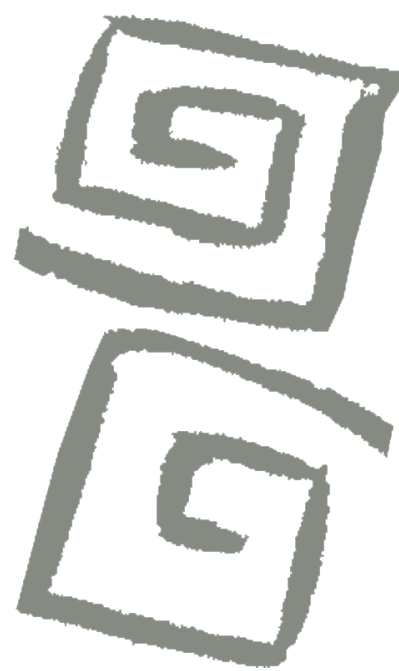

\title{
Salud mental y derechos humanos: La experiencia de los profesionales en formación en el uso de sujeciones mecánicas en Madrid, España
}

\author{
Mental health and human rights: The experience of \\ professionals in training with the use of mechanical \\ restraints in Madrid, Spain
}

Luis Nocete Navarro', Víctor López de Loma Osorio², María Fe Bravo Ortiz³, Alberto Fernández Liria ${ }^{4}$

${ }^{1}$ Autor de correspondencia. Psiquiatra. Unidad de Hospitalización Breve, Área de Gestión Clínica de Psiquiatría y Salud Mental, Hospital Universitario Príncipe de Asturias, Alcalá de Henares, España. $\square$ (iD)

${ }^{2}$ Residente de Psiquiatría. Área de Gestión Clínica de Psiquiatría y Salud Mental, Hospital Universitario Príncipe de Asturias, Alcalá de Henares, España. $\square$ (iD

${ }^{3}$ Psiquiatra. Jefa de Servicio de Psiquiatría y Salud Mental, Hospital Universitario La Paz, Universidad Autónoma de Madrid, Madrid, España.

$\triangle$ iD

${ }^{4}$ Psiquiatra. Profesor Honorífico, Universidad de Alcalá, Alcalá de Henares, España. $\bowtie$ iD
RESUMEN La sujeción mecánica en psiquiatría es un procedimiento permitido en España que despierta importantes conflictos éticos. Diversos estudios sostienen que su uso depende de factores no clínicos, como las experiencias de los profesionales y las influencias del contexto, más que de factores clínicos (diagnósticos o síntomas). El objetivo del estudio es comprender las experiencias de profesionales de salud mental en formación en relación con el uso de sujeciones en la red de salud mental de Madrid. Es un estudio cualitativo de tipo fenomenológico mediante grupos focales, realizados en 2017. Las entrevistas fueron transcritas para su discusión y análisis temático mediante Atlas.ti. Los resultados descriptivos sugieren que estas medidas producen malestar y conflicto con relación al rol de cuidador y se observan estrategias de adaptación a los mismos. A partir de los hallazgos, se reflexiona acerca de aspectos de sus experiencias y del contexto que influyen en su uso, así como de las contradicciones del cuidado en la práctica clínica.

PALABRAS CLAVES Salud Mental; Restricción Física; Inmovilización; Investigación Cualitativa; Derechos Humanos; Coerción; España.

ABSTRACT Mechanical restraint is a coercive procedure in psychiatry, which despite being permitted in Spain, raises significant ethical conflicts. Several studies argue that non-clinical factors - such as professionals' experiences and contextual influences - may play a more important role than clinical factors (diagnosis or symptoms) in determining how these measures are employed. The aim of this study is to understand how the experiences of mental health professionals in training relate to the use of mechanical restraints in Madrid's mental health network. Qualitative phenomenological research was conducted through focus groups in 2017. Interviews were transcribed for discussion and thematic analysis with Atlas.ti. Descriptive results suggest that these measures generate emotional distress and conflict with their role as caregivers. Our findings shed light on different factors related to their experiences and contexts that are important in understanding the use of mechanical restraint, as well as the contradictions of care in clinical practice.

KEY WORDS Mental Health; Physical Restraint; Immobilization; Qualitative Research; Human Rights; Coercion; Spain. 


\section{INTRODUCCIÓN}

\section{El contexto}

El empleo de sujeciones mecánicas y otras medidas coercitivas ha existido en nuestra disciplina desde su propio origen ${ }^{(1,2,3)}$. Desde un punto de vista conceptual, la definición del término sujeción mecánica varía entre diferentes autores. En castellano es habitual que se haga uso de los términos contención mecánica o inmovilización terapéutica indistintamente. En este estudio utilizaremos el término sujeción mecánica para referirnos a cualquier procedimiento que, mediante un dispositivo mecánico, limite la libertad de movimiento de una persona mediante la fijación de una o más partes de su cuerpo ${ }^{(4)}$. Descartamos el uso de los otros términos por considerarlos más ambiguos (se puede contener sin sujetar) o eufemísticos (se presupone una acción terapéutica).

La utilización de sujeciones mecánicas dentro de los contextos sanitarios está permitida en España. Sin embargo, al contrario que los ingresos involuntarios "por razón de trastorno psíquico" que se encuentran regulados por la ley ${ }^{(5)}$, la sujeción mecánica no cuenta con regulación por ningún precepto legal específico, y queda a cargo de ordenanzas locales y protocolos hospitalarios. La relevancia que ha adquirido la reflexión crítica en torno a las sujeción mecánica y la coerción en psiquiatría se ha puesto en relación con distintos factores ${ }^{(6,7)}$ : su elevada frecuencia ${ }^{(8,9)}$; su ubicuidad $^{(10,11,12,13,14,15)}$; la variabilidad de normativas que las regulan ${ }^{(16,17)}$; las controversias que supone su utilización ${ }^{(2,16,17,18,19)}$ en relación con el cumplimiento de los derechos humanos, la Convención de Derechos de las Personas con Discapacidad ${ }^{(20)}$ y las recomendaciones de organismos encargados de su defensa ${ }^{(20,21,22)}$, los conflictos éticos en la práctica clínica diaria ${ }^{(22,23,24,25)}$, la falta de evidencia sobre sus efectos terapéuticos ${ }^{(2,26,27)}$, las consecuencias dañinas sobre usuarios y profesionales ${ }^{(25,28,29,30,31,32,33)}$, el riesgo de abusos ${ }^{(21)}$; así como el auge y las presiones cada vez mayores por parte de los movimientos asociativos en primera persona ${ }^{(34)}$, cuando se conoce que existen alternativas ${ }^{(35)}$.
Esta controversia también se observa en nuestro país ${ }^{(34,36)}$. Sin embargo, a pesar de varios esfuerzos realizados para la transformación de los sistemas de salud mental ${ }^{(19,35)}$, su utilización continúa estando ampliamente extendida tanto en los países de nuestro entorno $^{(17,37,38)}$ como en España ${ }^{(17,39,40,41)}$.

En general, faltan estudios de calidad sobre la utilización de medidas coercitivas ${ }^{(18)}$ y hasta el momento no se pueden extraer conclusiones claras de su relación con las variables sociodemográficas (grupos de población determinados) o clínicas (trastornos o síntomas específicos). No obstante, diversos estudios subrayan la importancia de las experiencias de los profesionales y las características del contexto para comprender los factores relacionados con el uso de las sujeciones ${ }^{(42,43)}$. Algunos autores ${ }^{(17)}$ y organismos ${ }^{(44)}$ sugieren que dependen más de variables no clínicas -como la filosofía de las unidades ${ }^{(45)}$; los valores y hábitos ${ }^{(46)}$; las características de los centros $^{(8)}$ o los factores culturales, educacionales y organizacionales ${ }^{(42)}$ - que de cuestiones clínicas (diagnósticos, características de los usuarios).

Tras realizar una extensa búsqueda bibliográfica, encontramos que existen distintos trabajos cualitativos que indagan en las experiencias de los usuarios ${ }^{(47,48)}$, así como múltiples estudios cuantitativos acerca de la frecuencia, características demográficas y otros datos epidemiológicos ${ }^{(17,49)}$ en relación con la sujeción mecánica y otras medidas coercitivas, pero apenas se hallaron unos pocos acerca de las experiencias profesionales ${ }^{(50,51)}$. Especialmente, resulta Ilamativa la marcada ausencia de estudios con profesionales en formación ${ }^{(52)}$.

\section{Nuestra investigación}

A pesar de la creencia generalizada de que la coerción conlleva un daño a la libertad de las personas que la sufren y que, por tanto, debe considerarse un error, existe también una fuerte tendencia social a su justificación, considerándola necesaria para el funcionamiento correcto de nuestras sociedades ${ }^{(53)}$. 
Las situaciones (potencialmente) violentas sitúan a los profesionales ante el reto de dar una respuesta que permita garantizar la seguridad, sin olvidar la necesidad de soporte y cuidado que requieren los usuarios en un momento de máxima vulnerabilidad ${ }^{(54)}$. Por las consecuencias sobre los usuarios, se ha definido la sujeción mecánica como un procedimiento de riesgo ${ }^{(55,56)}$. Entre ellas se encontrarían secuelas emocionales (miedo, traumatización, impotencia, deshumanización) $)^{(55)}$, pero también físicas ${ }^{(57)} y$, en ocasiones, incluso mortales ${ }^{(19,32,58)}$. En relación con los profesionales, se ha sugerido que la relevancia de estudiar este fenómeno reside en que el impacto hacia ellos también es significativo ${ }^{(19,32,43)}$.

La investigación que presentamos tiene como objeto el estudio de la experiencia de los profesionales de salud mental en formación (residentes) con relación a las sujeción mecánica. Con experiencia, debe entenderse aquel conocimiento que se deriva o adquiere por haber vivido una situación o circunstancia ${ }^{(59)}$, un modo de conocimiento basado en lo vivido. Las preguntas que han guiado nuestra investigación son: 1) ¿cómo describen los profesionales de salud mental en formación su experiencia en relación con la decisión de usar y ejecutar sujeción mecánica?; y 2) ¿qué aspectos de esta experiencia y de qué manera influyen en los profesionales a la hora de decidir el uso de sujeciones mecánicas? Resultados preliminares de esta investigación, anteriores a la elaboracion de la teoría fundamentada, pueden encontrarse en un cuaderno téncnico de la Asociación Española de Neuropsiquiatría ${ }^{(60)}$.

\section{METODOLOGÍA}

\section{Diseño del estudio y marco teórico}

Para dar respuesta a estas preguntas de la manera más adecuada, adoptamos una metodología cualitativa mediante grupos focales. La metodología cualitativa parte del supuesto de que la realidad y el conocimiento son múltiples y complejos, que están influidos por el contexto y los valores socioculturales y que lo que las personas piensan, sienten y hacen conforma también la realidad ${ }^{(61,62)}$. La elección de este enfoque nos da la posibilidad, a través del diálogo, la interacción y el análisis del discurso, de comprender las experiencias desde la perspectiva de las personas que experimentan el fenómeno de las sujeciones, dentro del marco temporal y contexto sociocultural naturales en que se encuentran ${ }^{(63)}$. La aproximación a la experiencia se realiza desde una perspectiva fenomenológica, que pone el énfasis en cómo el mundo se hace presente en la subjetividad de los participantes y en los significados que se dan, en sus propios términos ${ }^{(64)}$. Nuestro método es de tipo hermenéutico, porque trabajamos a partir de una teoría de interpretación que es especialmente útil para generar hipótesis que den sentido a una cuestión compleja y conflictiva, y sobre la que existe poca investigación ${ }^{(65)}$. La recogida de datos se realizó mediante grupos focales, que permiten a las participantes la posibilidad de expresar sus ideas y opiniones libremente ${ }^{(66)}$, a través de las interacciones y la puesta en común de actitudes, puntos de vista y discursos de cada participante, apoyando la exploración, clarificación y profundización de las aportaciones individuales. Las interacciones involucran una escucha y observación atentas, en los que el conocimiento emerge del intercambio entre la subjetividad de los investigadores, el contexto y el objeto investigado ${ }^{(64)}$.

\section{Estrategia de selección y tipo de muestreo}

La estrategia de selección de participantes fue intencionada y razonada. Los participantes se eligieron en función de la representatividad y variabilidad de los discursos existentes en la población y no se basaron en probabilidad estadística. La selección se llevó a cabo de la siguiente manera: 1) se definieron perfiles con características determinadas (en función de la experiencia propia del equipo investigador, los expertos consultados y la bibliografía revisada) para conseguir grupos específicos relevantes que representaran la estructura de 
la población de referencia (muestra estructural); 2) la selección se realizó a través de informantes claves que conocían a profesionales con las características que buscábamos $y$, una vez localizados, se facilitó la hoja informativa y el consentimiento informado; 3 ) cuando se alcanzó un número adecuado, se procedió a su distribución en vistas de favorecer la riqueza y heterogeneidad discursiva. Durante la selección se intentó evitar al máximo que los participantes tuviesen una relación cercana entre sí o con el equipo. Sin embargo, resultó irremediable que algunos de los participantes hubieran compartido previamente otros espacios, dado el ámbito reducido de la población de referencia y la existencia de lugares comunes de formación fuera del ámbito laboral. En los pocos casos en los que coincidieron personas que se conocían previamente, se aseguró que la relación personal entre ellos no fuera estrecha y que su participación no se viese coartada por la presencia del otro miembro.

Como criterios generales de inclusión, se consideró que fuesen profesionales de salud mental en formación, que trabajasen en la red pública sanitaria de la Comunidad de Madrid y que quisieran participar de manera voluntaria. Como hemos señalado, para que las experiencias de los participantes fueran lo más parecidas posible a las que existen en la población natural de la que provienen, se definieron unos perfiles que posteriormente se tomaron como referencia en la selección de la muestra. En la creación de estos perfiles se tuvieron en cuenta distintas variables que dividían a la población y que se estimó que tenían una influencia significativa sobre el fenómeno de estudio.

\section{La categoría profesional}

Se dividió a la población en función de la profesión de origen: enfermería (EIR), psicología (PIR) y medicina (MIR). Si bien los psicólogos clínicos están en menor contacto directo con el uso de sujeciones mecánicas durante su formación y desarrollo profesional (no suelen participar en la indicación ni en el procedimiento), su implicación en el paradigma que sustenta estas prácticas es igualmente importante. Por ello se buscó una participación equilibrada de todas las categorías en los grupos. Por otro lado, y al contrario que los profesionales especializados, los residentes no están asignados a ninguna unidad o dispositivo concreto. Sin embargo, a lo largo de su formación y, generalmente, al inicio, todos pasan por espacios en los que el uso de las sujeciones se da con mayor o menor frecuencia. Por ello, en este caso, no ha sido necesario clasificar a los profesionales según el lugar de trabajo.

\section{El área hospitalaria o la unidad docente de formación}

Es conocido por los profesionales de salud mental de la región de Madrid que el modelo formativo difiere, en parte, en función de la filosofía o la cultura de trabajo que existe en cada unidad docente. Así, pueden encontrarse áreas con una tendencia hacia teorías y prácticas más cercanas a lo social o comunitario y a lo psicoterapéutico, y otras más cercanas a posicionamientos más médicos, biologicistas y hegemónicos, y que suelen estar relacionados con posturas más o menos críticas con el uso de medidas coercitivas en salud mental. Sin embargo, esto no es garantía de que los profesionales en formación en las distintas áreas compartan la posición general de rechazo/apoyo al uso de sujeciones mecánicas. Por ello, se dividieron en tres perfiles según la actitud que se presume que tengan hacia estas medidas: crítica ("no deberían utilizarse"), pragmática o neutral (" a veces es necesaria por seguridad") o positiva/terapéutica ("se necesitan para el tratamiento")(67). Para intentar garantizar que el perfil sea el buscado dentro de cada área, nos apoyamos en los informantes. Finalmente se incluyeron residentes de nueve de las 22 unidades docentes.

\section{Otras características: género, y tiempo de formación}

Las cuestiones relacionadas con el género y los años de desarrollo profesional ${ }^{(19,68)}$ pueden influir en las vivencias de los participantes, por 
lo que se tuvieron en cuenta para que la muestra representara las diferencias poblacionales.

A partir de estas variables, se incluyeron participantes que cumpliesen con un abanico de características lo más amplio posible, de forma que en nuestros grupos estuvieran "representadas", con la mayor fidelidad, las distintas experiencias y discursos de los profesionales de salud mental en formación. En un inicio el número de participantes y grupos se dejó abierto. A medida que se desarrollaron los primeros grupos, se realizó un análisis preliminar de los datos, a partir del cual se redefinieron los participantes posteriores, con el objetivo de encontrar perfiles y datos que no habían aparecido, lograr la saturación teórica y refinar la teoría emergente (muestreo teórico). Se dejó de reclutar a los participantes una vez que se llegó a la saturación conceptual, si bien esta nunca se completa, estuvo limitada por el tiempo y los recursos.

\section{Descripción de la población y marco espacial de la investigación}

El estudio incluyó a 21 residentes, distribuidos en tres grupos focales diferentes de siete personas. Un residente rechazó su participación antes de iniciar el grupo aludiendo dificultades con el horario, y pudo sustituirse. El día del encuentro de los grupos, dos participantes no pudieron acudir, por lo que el número final total fue de 19 residentes: siete de psiquiatría (MIR), seis de enfermería especializada en salud mental (EIR) y seis de psicología clínica (PIR). El total de participantes estaba conformado por doce mujeres y siete hombres, de distintos años de formación (algunos participantes estaban comenzando la especialización, otros se encontraban en pleno proceso formativo y otros al final de su especialización). En un inicio se incluyeron participantes que pertenecían a once hospitales públicos de la Comunidad de Madrid, pero con la pérdida de dos participantes finalmente quedaron nueve áreas sanitarias, sin que ello medrase la representación de las distintas posturas teóricas mayoritarias en función de los perfiles de sus unidades docentes. Durante el periodo formativo todos los residentes realizan rotaciones por unidades en las que se está en contacto con la sujeción mecánica y la toma de decisiones que conlleva.

\section{Recogida de los datos}

Los grupos focales se efectuaron durante el año 2017. Cada encuentro tuvo una duración aproximada de una hora y media, y se realizaron sobre la base de un guion semiestructurado, desarrollado por el equipo investigador y supervisado por el investigador con mayor experiencia. El guion no funcionó como un cuestionario rígido, sino como un marco flexible para explorar áreas de interés, utilizando preguntas abiertas para obtener respuestas libres, así como preguntas enfocadas puntuales para obtener respuestas singulares y útiles. El guion sirvió, además, como elemento homogeneizador de las intervenciones de los distintos moderadores, incluyendo instrucciones acordadas previamente por el equipo sobre el desarrollo de la entrevista. Los temas más importantes se centraban en las experiencias de los profesionales con la sujeción mecánica (actitudes, pensamientos, emociones y acciones). Los encuentros grupales se realizaron fuera del entorno sanitario, en un lugar tranquilo, y se generó un ambiente distendido sin presiones institucionales. Antes de su inicio, se volvió a explicar la naturaleza del estudio y a solicitar el consentimiento informado, se verificó que la información se entendía y que la participación era voluntaria. A continuación, se dieron instrucciones para favorecer el correcto desarrollo del grupo, recordando que podían expresarse y dialogar libremente, que no se buscaba el acuerdo. Durante los grupos, la conversación sucedió de forma fluida y dinámica, existiendo una interacción adecuada entre los participantes. Fueron conducidos por una persona que moderaba, responsable de favorecer y guiar la participación y elaboración del discurso, y una que observaba que, sin interferir en el desarrollo natural de la entrevista, registraba y hacía anotaciones sobre lo que sucedía mientras grababa el contenido de las mismas en audio y vídeo. 


\section{Análisis de los datos}

Nos guiamos por las recomendaciones de Berenguera et al. ${ }^{(61)}$ y por la lectura constructivista que hace Charmaz de la teoría fundamentada de Glaser y Strauss ${ }^{(69,70)}$. Nuestro análisis fue un proceso progresivo que va desde la descripción de los datos, pasa por la construcción y el ordenamiento de conceptos/categorías, y finaliza en la teorización ( $\mathrm{Fi}$ gura 1). Las grabaciones de las intervenciones fueron transcritas de manera literal por los investigadores, añadiendo anotaciones acerca de la información no verbal. No se transcribieron los nombres de los informantes, sino que se asignaron códigos alfanuméricos. Se unificó el contenido de las transcripciones en un único corpus textual para facilitar la lectura y análisis, que fue leído varias veces por todos los investigadores para familiarizarse con los datos en bruto. En las relecturas, se realizan anotaciones preliminares sobre posibles códigos y categorías temáticas, discutiendo este ejercicio entre todos, a partir de las primeras intuiciones e ideas ("naive reading") ${ }^{(71)}$. Se utiliza el programa de análisis de datos cualitativos Atlas.ti versión 1.6.0. para MAC como herramienta auxiliar para el etiquetado y la codificación. En función de sus propiedades, se otorgó una codificación inicial mediante la fragmentación del texto en citas (líneas o párrafos) que funcionan como una unidad de sentido sobre algún aspecto de su experiencia. Los primeros códigos son descriptivos, ligados al contenido literal de la cita. El proceso de codificación se sofistica durante todo el análisis mediante la lectura iterativa y detallada de lo que va emergiendo $^{(72)}$. A través del método comparativo constante, y la interpretación y abstracción reiterativa, se buscan las relaciones entre los códigos para refinarlos y agruparlos en categorías conceptuales, que integran categorías más descriptivas hasta llegar a categorías
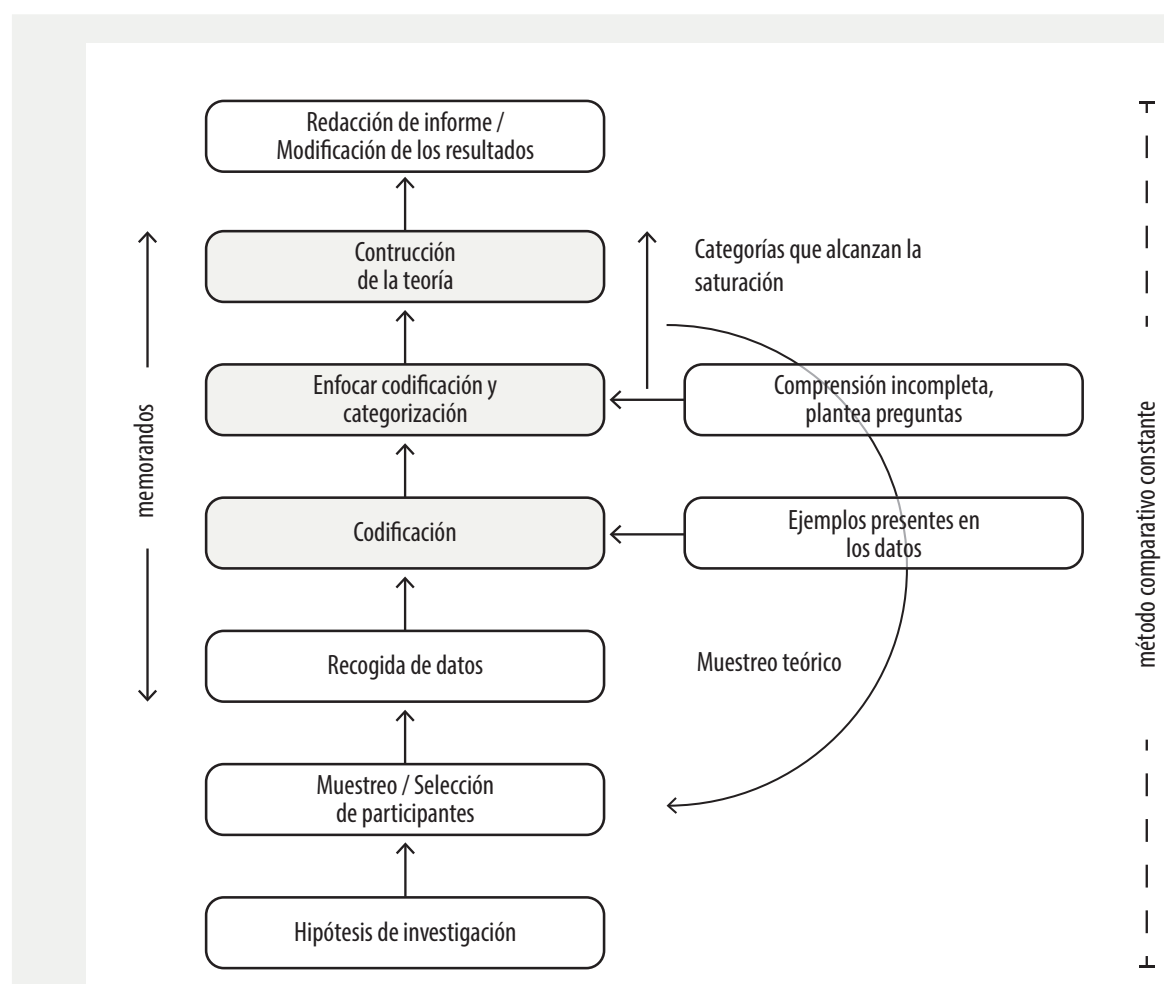

Figura 1. Representación visual de la metodología de la Teoría Fundamentada.

Fuente: Elaboración propia a partir de Tweed y Charmaz $^{(70)}$ 
centrales y subcategorías (codificación axial) cuando la saturación de los datos fue suficientemente adecuada. Por último, tiene lugar la codificación teórica, relacionando los resultados con las hipótesis en función de su relación con los datos, con el objetivo de comprender los significados emergentes ${ }^{(72)}$. Durante todo el proceso, se contrasta la categorización y codificación con el contenido, y se verifica que la teoría emergente tenga su base en lo que las personas contaron, en un esquema lógico, sistemático y explicativo de los conceptos construidos.

\section{Estrategias para garantizar la calidad y rigor de la investigación}

Cómo evaluar la calidad de las investigaciones cualitativas es aún objeto de debate importante $^{(73)}$. Para garantizar la calidad de nuestra investigación, se siguieron las propuestas de varios autores $^{(61,74,75)}$. Se ha hecho un esfuerzo por ilustrar la adecuación metodológica de los objetivos, metodología y método, pudiéndose realizar un seguimiento detallado de la rigurosidad del diseño, de los pasos tomados, manteniendo un compromiso de transparencia. Para aumentar la fiabilidad y validez del estudio se realizó un muestreo y análisis riguroso, empleando técnicas de triangulación para verificar los resultados y su representatividad: triangulación de datos, de investigadores, y de teorías. Asimismo, se ha mantenido una actitud crítica, cuidadosa y reflexiva sobre la construcción de nuestra investigación, tanto al objeto de estudio, como hacia nuestra propia subjetividad como investigadores.

\section{Aspectos éticos}

Todos los participantes recibieron la información sobre el estudio y el consentimiento informado oral y escrito, y aceptaron participar de manera voluntaria y anónima. Conocían que eran libres de abandonarlo en cualquier momento y que no existía ningún tipo de compensación por su inclusión. Para garantizar el cumplimiento de los requisitos éticos exigibles, se solicitó la evaluación del Comité de Ética de la Investigación del Hospital Universitario La Paz (código HULP: PI-2928), que fue aprobada en octubre de 2017. Los sujetos fueron seleccionados de forma justa, sin discriminación entre los que cumplían los requisitos buscados. Se tuvieron en cuenta los posibles riesgos relacionados con la divulgación de su participación, extremándose el anonimato, la intimidad y la confidencialidad de datos y participantes ${ }^{(76)}$. El tratamiento y la comunicación de datos se ajustó a la normativa local ${ }^{(77)}$ y derechos A.R.C.O., así como a los principios de la Declaración de Helsinki ${ }^{(78)}$.

\section{RESULTADOS}

En el análisis de los datos se identificaron 978 códigos in vivo, puramente descriptivos. Tras ordenarlos en categorías conceptuales más abstractas, se obtuvieron un total de 86 códigos, que se presentan en tres grandes categorías mayores de significado en función del objeto de la experiencia, en combinación con otras tres categorías en función del origen de la experiencia (Figura 2).

Dentro de este esquema, se organizan el resto de subcategorías y los distintos códigos que dan respuesta a la primera pregunta de investigación. Para este trabajo, los resultados se desarrollarán, sin embargo, en función del análisis posterior, que sustenta las bases para una teoría fundamentada acerca del uso de sujeción mecánica en profesionales en formación, dando respuesta a la segunda pregunta de investigación acerca de los distintos aspectos de la experiencia y la manera en que influyen a la hora de indicar y usar sujeción mecánica. Se exponen en base a tres grandes categorías temáticas: A. Experiencias que anteceden a la sujeción mecánica, B. Experiencias durante la indicación de sujeción mecánica y C. Experiencias tras el uso de sujeción mecánica.

En la Figura 3, se refleja de forma gráfica la teoría fundamentada. Los significados que subyacen a los códigos y categorías se 


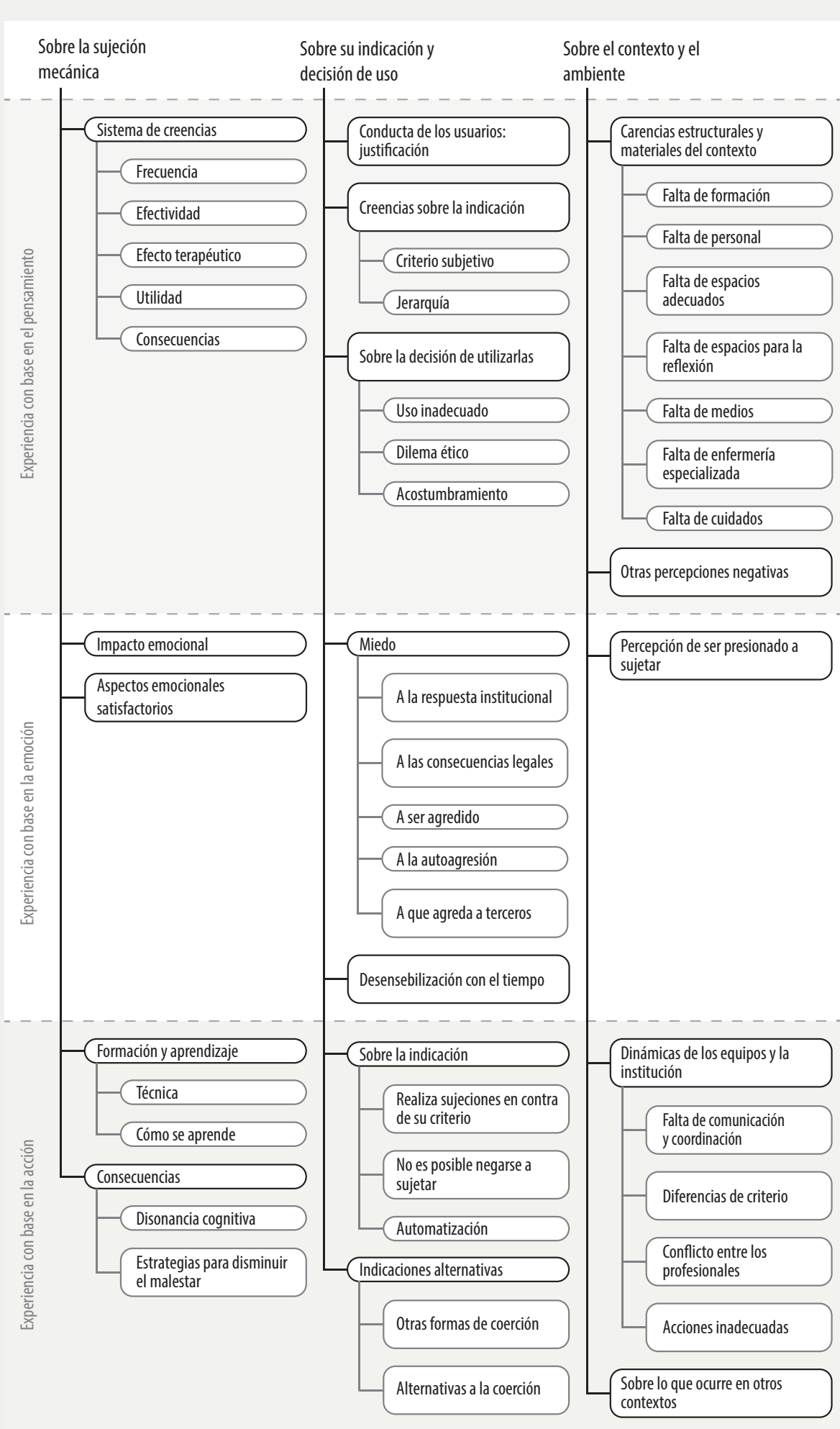

Figura 2. Principales categorías, subcategorías y códigos de la experiencia de los profesionales.

Fuente: Elaboración propia. 


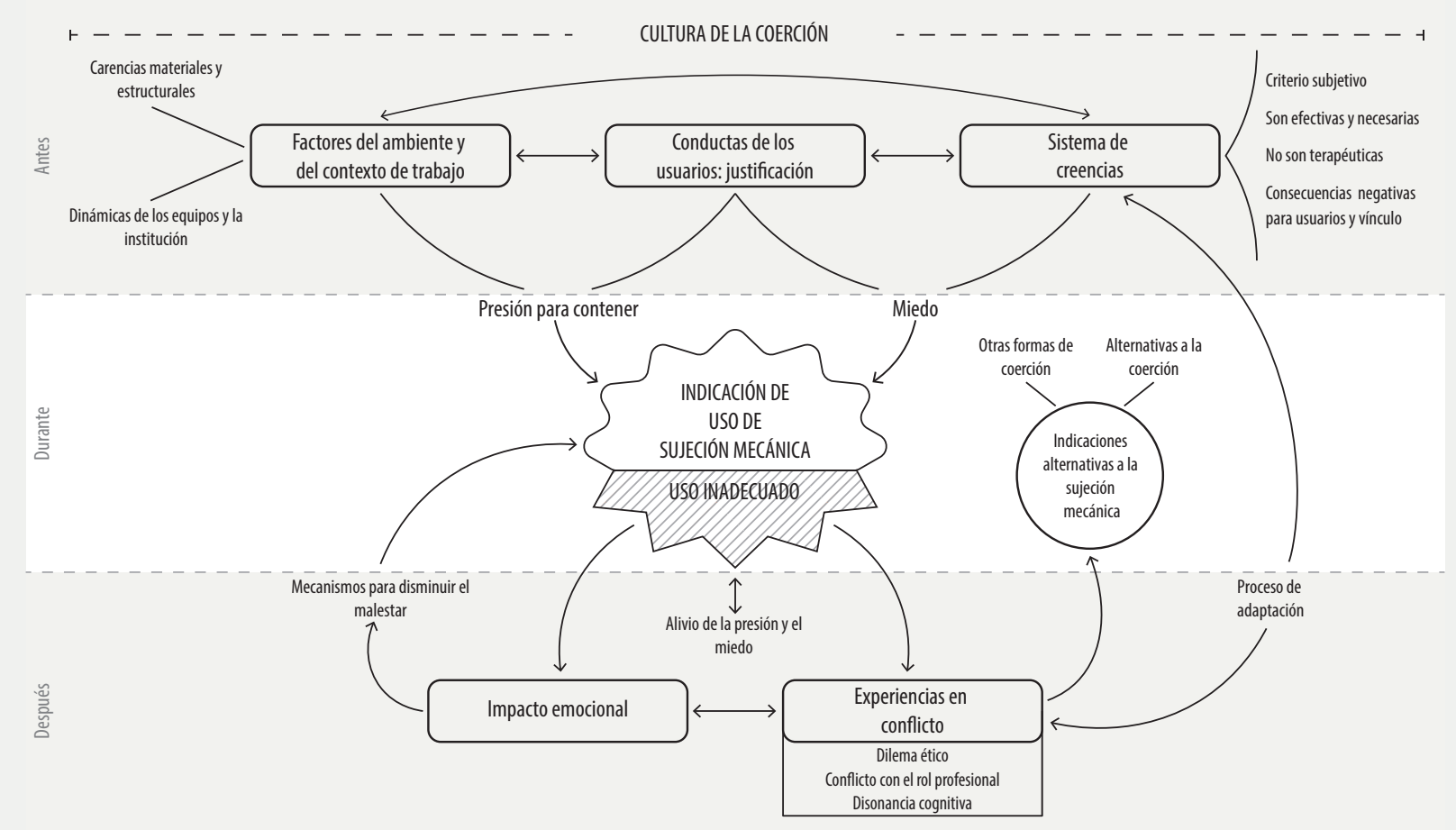

Figura 3. Teoría fundamentada de la experiencia de los residentes de salud mental en el proceso de decisión e indicación de sujeción mecánica.

Fuente: Elaboración propia.

sostienen en los extractos del discurso (citas), y es desde donde se construye sentido de los mismos (Tablas 1 , Tabla 2 y Tabla 3).

\section{Experiencias que anteceden a la sujeción mecánica}

\section{Factores del ambiente y el contexto de trabajo}

De manera generalizada, los profesionales aluden a una serie de circunstancias del contexto y el ambiente de trabajo cuando hablan de la sujeción mecánica. Destacan la importancia de los espacios, la estructura y los medios materiales, pero también las interacciones entre profesionales, las dinámicas de equipos y el funcionamiento institucional, y consideran que su influencia es determinante en la toma de decisiones sobre la sujeción mecánica.

\section{Carencias estructurales y materiales}

Cuando se refieren a las condiciones materiales y estructurales en que trabajan, las relacionan con un mayor uso de sujeciones mecánicas. Enumeran una serie de carencias que, en su experiencia, dificultan la realización de acciones alternativas y favorecen la indicación de sujeción mecánica: falta de personal en los equipos, profesionales no especializados (enfermeros, auxiliares y otros, que trabajan en salud mental), y formación precaria tanto en las contenciones como en sus alternativas. Perciben la falta de medios materiales, financiación o tiempo, que los espacios de atención son inadecuados a las necesidades de los usuarios, que no existen momentos formales en los que se reflexione acerca de los incidentes que terminan con una sujeción mecánica, y que todo ello contribuye a su utilización. 
Tabla 1. Experiencias que anteceden a la sujeción mecánica. Madrid, España, 2017.

Categorías / subcategoría Datos (citas)

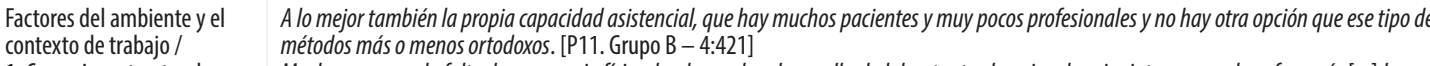

1. Carencias estructurales y Muchas veces, es la falta de un espacio físico donde puedan desarrollar la labor tanto el equipo de psiquiatras como de enfermería [...] la

materiales

planta de psiquiatría [...] no está adaptada para el paciente psiquiátrico. / Muchas veces, ya una vez se ha construido el hospital, se dice: "hay que poner la planta de psiquiatría", y se abre la unidad de psiquiatría sin tomar en cuenta las peculiaridades del paciente... Como, por ejemplo, cuando se abre una unidad de cuidados intensivos sí que se piensan dónde estaría el ventilador, el respirador... ino?... Que esté cerca de un quirófano... [E16. Grupo C - 4:859/1059-60]

Factores del ambiente y el contexto de trabajo / 2. Dinámicas de los equiposy la institución

Yo síme he sentido presionado muchas veces para contenciones, tanto por parte de gente de la propia Urgencia, por parte de enfermería [...] y por parte de mi propio servicio. Esa presión muchas veces deriva en una SM, especialmente cuando es por parte del propio servicio, cuando es

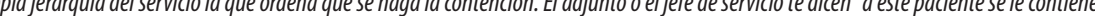
después de haber contado tú que se podría mantener al paciente de otra forma y tal... [Se hace] "contención" en cuanto el adjunto lo ordena. De alguna manera si no eres tú el que se expone a una falta disciplinaria o lo que sea en caso en que eso no sea así, broncas... cualquier cosa entre medias. Y eso ha sucedido, yo lo he vivido en mis carnes, compañeros mios lo han vivido en sus carnes, quiero decir, es algo que ahí está. [A1. Grupo A - 4:78]

[...] yo creo que el paciente psiquiátrico en la Urgencia [...] es un paciente de segunda... Que molesta, que es un paciente que no se le tiene la misma consideración, que sele deja relegado y que fastidia. Hay mucha incomprensión, yo creo. No se le da un trato (bueno) por parte también de los médicos generales: "este está chillando a ver cuándo subis a la planta". Como un lenguaje muy despectivo, más estigmatizante, y por parte de casi todo el personal también. [A4. Grupo A - 4:72]

Sistema de creencias

Yo creo que las contenciones, el problema que tienen es que son tremendamente efectivas, cumplen su función que es inmovilizar, que es que la persona no lleve a cabo lo que sea que quisiera hacer, lo que fuera... no lo llevan a cabo. [...] Se hacen porque son 100\% efectivas: sujetan. [A1. Grupo A - 4:747]

Yo creo que produce un daño a la dignidad de la persona. Decirte, literalmente, que le están arruinando la vida. [P17. Grupo C - 4:1165]

Conductas de los usuarios (justificación consciente del uso de sujeciones)

Cultura de la coerción

Fuente: Elaboración propia
Ese famoso riesgo de fuga en pacientes que están entre comillas esperando cama en la planta, pero que debería estar ingresados involuntariamente... pues es un riesgo de fuga. Muchas veces se dice que si lo pudiéramos subir a la planta no lo tendríamos que estar conteniendo. [A1. Grupo A - 4:145]

Ella busca la cama, claro. Ella quiere estar en una cama. Entonces... bueno, pues se le podría efectivamente poner en la cama sin contenerla, pero se la contiene. Yya, pues en cierto modo, como castigo. Pues se la contiene como castigo, claro. [M19. Grupo C - 4:1185]

Yo nunca he visto una valoración judicial que lleve la contraria al psiquiatra, sólo una vez por una circunstancia especial. Aun estando seguro de que al menos en mi hospital hemos hecho IT que no estaban justificadas para nada. [M13. Grupo B - 4:327]

También es el paternalismo de "soy el responsable de todas las conductas" y hasta dónde llega. [A4. Grupo A - 4:131]

Lo que no entiendo muy bien es cómo el tener más recursos va a limitar, teniendo en cuenta los criterios que tenemos ahora de contenciones, esas contenciones. Igual limita que atemos a los pacientes a la cama, pero habrá que contenerles de otra manera. [M19. Grupo C - 4:1042]

\section{Tabla 2. Experiencias durante la sujeción mecánica. Madrid, España, 2017.}

Categorías/subcategorías Datos (citas)

Percepción de ser presionado a sujetar: atrapado en demandas contradictorias

Miedo / 1. A que"pueda pasar algo"

Miedo / 2. A la autoagresión

Miedo / 3. A las consecuencias legales

Miedo / 4. A ser agredido

Miedo / 5. A la respuesta institucional

Uso inadecuado de sujeción mecánica

Indicaciones alternativas a la sujeción mecánica

Fuente: Elaboración propia.
A míme han echado broncas porque yo hellegado a dejar pacientes psicóticos sin contener, e irme... y me he comido la bronca. (M13: yo también) / A mí en la planta a veces me presionan para que haga contenciones. [M7. Grupo B - 4:319/357]

Es que en la observación general a la mínima ya te piden contención porque, por ejemplo, hay un señor de 90 años con EPOC al lado... y te dicen que "es que se pone nervioso", aunque el paciente no esté haciendo nada, como mucho quiere irse... [M8. Grupo B - 4:357]

A mí, por ejemplo, me genera conflicto el paciente que tiene claro criterio de ingreso, pues lo que decía A, un psicótico con descompensación en la Urgencia, pero que está tranquilo, pero que el riesgo ese de imprevisibilidad, que claro no es algo tangible, la imprevisibilidad. Dices, joder, ¿le contienes o no? [M14. Grupo C - 4:983]

De las que he visto y de las que he indicado, alguna quizá sí que era terapéutica. Pues eso, casos en los que lo ves como súper claro, ¿no? Casos como de... "te vas a abrir la cabeza y no hay manera de que no te vayas a abrir la cabeza", pues ya está. [M13. Grupo B - 4:426]

Además, yo creo que los adjuntos, al menos los nuestros, actúan más con miedo a la responsabilidad legal o las consecuencias, que porque la IT esté indicada o no según el protocolo. De largo, creo que el factor que más influye es el miedo a que esa persona que está psicótica tranquila se escape y le atropelle un autobús en la castellana y la responsabilidad legal que pueda tener sobre la guardia. [M13. Grupo B - 4:288]

La sensación de peligro inminente. A mí me ha pasado que me estén lanzando objetos. [M7. Grupo B - 4:42]

...entonces, desde ahi está justificado: a ti nadie te va a echar la bronca por inmovilizar, en la urgencia; ahora, sí te puedes llevar un broncón importante por no hacerlo. / Yo estoy de acuerdo con lo que él dice: te pueden echar la bronca por no hacerlo, pero nunca te van a decir muy mal por haberlo hecho, eso no lo he visto en el tiempo que llevo. [M13. M8. Grupo B - 4:378/80]

...en mi hospital pasa lo mismo: que yo creo que hay muchas, sobre todo en la urgencia, y bueno, en la planta yo creo que se podrían evitar algunas de las que se hacen, pero en el caso de la urgencia repito lo que ya he dicho antes, que yo creo que habría que poner alternativas a eso. [M7. Grupo B - 4:386]

Bueno sí, si hablamos de cambiar, del paciente, de atarle a una cama a encerrarle en una habitación, pues claro que se podría hacer ese cambio si tuviésemos muchas habitaciones donde encerrar a pacientes. [M19. Grupo C - 4:1050]

Una vez que colaboramos nosotros en la docencia y esto sí que salió fue por ejemplo una solución que se nos dio fue preguntarle a la persona quién quiere que esté con él, y dejar que esa persona que él quiere, esté con él. [P12. Grupo B - 4:314] 
Tabla 3. Experiencias tras la sujeción mecánica. Madrid, España, 2017.

\begin{tabular}{|c|c|}
\hline ategorías / subcategoría & Datos (citas) \\
\hline Impacto emocional & $\begin{array}{l}\text { No es nada agradable... para nada. Son momentos en los que luego te planteas si verdaderamente quieres seguir haciendo esto. Porque se hace } \\
\text { bastante duro... [A3. Grupo A - 4:20] } \\
\text { Yhe de decir que me pasémucho tiempo sin poder hacerlo yo sola [...] Porque necesitaba que alguien estuviera ahí, por si pasaba algo. No sé, } \\
\text { pensaba: "esto va a ser tan traumático para todos..." [A2. Grupo A - 4:646] }\end{array}$ \\
\hline $\begin{array}{l}\text { Alivio de } \\
\text { miedo }\end{array}$ & $\begin{array}{l}\text { Claro, es que cuando tú eres conservador a la hora de poner las contenciones, es cuando te pueden venir los problemas. Si tú } \\
\text { contenciones a diestro y siniestro, no tienes problemas... / Te evitas problemas de un lado / Eso es así. [M19. M14. Grupo C }\end{array}$ \\
\hline $\begin{array}{l}\text { Experiencias e } \\
\text { 2. Dilema étic }\end{array}$ & $\begin{array}{l}\text { Yo por norma general, me siento muy rabiosa y muy deshonesta, conmigo, hacia los valores que yo tengo, las ideas que yo tengo, y hacia la } \\
\text { persona contenida. [E15. Grupo C - 4:1092] } \\
\text { A mí, mientras [...] me viene que falta toda la parte ética y moral. ¿Hasta dónde es ético y moral hacer todo esto? [...] Y si también deberíamos } \\
\text { tener un poco de miedo no solo a lo que nos puede pasar legalmente, sino a las consecuencias morales o éticas de lo que estamos haciendo con } \\
\text { la vida de otros... algo tan básico como es su libertad. A mí, de las contenciones mecánicas, es la parte sin lugar a dudas que más dudas me } \\
\text { genera. [P11. Grupo B - 4:295-7] }\end{array}$ \\
\hline $\begin{array}{l}\text { Procesos de adaptación a la } \\
\text { experiencia conflictiva }\end{array}$ & $\begin{array}{l}\text { Yo creo que toda esa parte ética y moral conforme vas avanzando más en la residencia no es que se te olvide más, pero como que el proceso de } \\
\text { indicar una inmovilización terapéutica yo no sé si lo vas automatizando, o si es una defensa tuya, en plan... para no sentirte... Porque yo me } \\
\text { sentí realmente mal de R1 [primer año de residencia] y ahora, no me hace ni mucho menos gracia, pero no me siento lo mal que me sentía de } \\
\text { R1. [...] El primer día era cómo: "qué horror, lo voy a pasar fatal cada vez", y la verdad es que ahora, tristemente, no lo pasas igual de mal que al } \\
\text { principio. [M13. Grupo B - 4:300-519] }\end{array}$ \\
\hline $\begin{array}{l}\text { Estrategias y mecanismos } \\
\text { para aliviar el malestar que } \\
\text { les producen las sujeciones }\end{array}$ & $\begin{array}{l}\text { A mí la teoría me ayuda mucho. Ponerme más filosófico me ayuda a procesarlo y colocarme en un rol casi de poder decir que hay una parte que } \\
\text { no es responsabilidad mía, hay una parte que no soy yo el agente ejecutor } 100 \% \text {, yo estoy ejecutando la mano invisible del Estado, o la mano } \\
\text { ejecutora de lo social, lo social me ha dispuesto igual que ha dispuesto al policía. [A1. Grupo A - 4:630] } \\
\text { Racionalizamos, normalizamos [...] Pero eres consciente de que... te haces pequeño para justificar que no puedes cambiar semejante sistema. } \\
\text { [...] Es que me quedo en "esto es de locos", nunca mejor dicho, pero... Yo por lo menos tiro por esa parte, evoluciono a normalizar lo no }\end{array}$ \\
\hline
\end{tabular}

Fuente: Elaboración propia.

\section{Dinámicas de los equipos y la institución}

Para los residentes, existen discrepancias sobre las medidas coercitivas entre psiquiatras y enfermeros, así como una falta de coordinación y comunicación en el contexto de la sujeción mecánica, lo que facilita la aparición de conflictos por las normas y estas medidas. De hecho, se señala que uno de los motivos para ejecutar una sujeción es evitar el conflicto entre el personal. Sin embargo, también perciben la existencia de una jerarquía a la hora de decidir sobre la sujeción mecánica, en la que prima, en última instancia, el criterio de los psiquiatras facultativos. Cuando hay desacuerdo, la jerarquía se vive tanto con frustración, como con alivio por no tener que asumir la responsabilidad de una decisión que consideran difícil. Además, describen que en los equipos se dan una serie de actitudes y acciones que pueden desembocar en conflictos que acaben justificando una sujeción mecánica: engaños, dar mensajes incoherentes (contradicción entre turnos), actitudes impositivas y falta de diálogo, gritar y amenazar, etc. Esto es algo que afecta también a profesionales que, siendo de otras especialidades, atienden a personas con sufrimiento mental, fundamentalmente en la Urgencia. En ellos perciben, además, falta de interés o de colaboración, un lenguaje o una actitud despectivos y un trato desigual hacia las personas con sufrimiento psíquico. Por último, con relación a la institución, piensan que existe poco apoyo para implementar medidas alternativas y que sería necesario un cambio de cultura y de organización. 


\section{Sistema de creencias de los profesionales}

De las experiencias de los residentes se revelan sus creencias acerca de la sujeción mecánica. Consideran que son mecanismos que "funcionan", que son "efectivas" pues cumplen con el objetivo para el que se diseñaron. Aunque algunos reconocen que en otros lugares se han eliminado, la mayoría la ven como una medida que no puede (incluso que no debe) eliminarse, expresando desconfianza y recelo ante la posibilidad de una atención libre de sujeción mecánica, que consideran necesaria para garantizar la seguridad. No obstante, de forma mayoritaria estiman que la sujeción mecánica no es terapéutica en sí misma. Varios de ellos consideran necesario especificar en qué sentido es efectiva o terapéutica, por considerar que son términos ambiguos e interpretables. Expresan, por ejemplo, que podrían tener un efecto terapéutico indirecto cuando sirven para evitar el riesgo de un daño físico, viendo en ese caso la sujeción mecánica como una medida de protección.

Resulta llamativo que, a pesar de considerarla efectiva, necesaria, e incluso terapéutica, al poner el foco sobre sus consecuencias en las personas que la sufren, reconocen que el impacto es por lo general negativo, describiendo daños a nivel psicológico, físico, moral o conductual, incluso tan solo con presenciar la sujeción mecánica sobre otros. En su experiencia, este daño también puede darse en el vínculo terapéutico, que puede hasta romperse. Algunos lo asocian con las condiciones en que se realizó la sujeción mecánica, las características previas de la relación entre usuario y profesional y otros factores. Enfermería vive especialmente de cerca esta ruptura de la relación terapéutica.

En último lugar, una experiencia especialmente repetida fue la de sentir que, a pesar de que se intentan establecer criterios determinados para la aplicación de sujeción mecánica, son finalmente decisiones personales y subjetivas, las que motivan su indicación. Aunque se apela en ocasiones al sentido común, los criterios de facto aparecen como interpretables, con elevada variabilidad inter e intrapersonal (por sus emociones, creencias, etc.) o de otras variables externas. Además, encuentran que existe una tendencia personal a aplicarlas o no. Los residentes expresan que muchas veces no llegan a tener en claro el porqué de la indicación de la sujeción mecánica, los criterios u objetivos en que se basan. Refieren que los protocolos son ambiguos y por ello no son de ayuda o guía en las indicaciones.

\section{Sistema de creencias colectivo: la cultura de la coerción}

Del análisis del discurso emerge una categoría transversal que hemos denominado cultura de la coerción. La cultura de la coerción puede entenderse como el conjunto de supuestos que estructuran la institución, los equipos y los profesionales, que define sus tareas y organización, y que opera de forma constante, sea uno o no consciente de ello, conformando lo que es y no es posible hacer dentro de los determinantes que impone el propio contexto social/grupal/institucional. Este sistema de creencias colectivo, acaba interiorizándose, de modo que permea las acciones, pensamientos y emociones de los profesionales en el desarrollo de su práctica. Según lo referido, algunos de los elementos comunes que caracterizan la cultura de los servicios de psiquiatría son: el predominio del modelo biomédico y del discurso de la gestión del riesgo, el paternalismo, la apelación a la norma y la autoridad (jerarquía) en el tratamiento, o la naturalización de la coacción como parte del trabajo. Estos elementos se ven reflejados de manera transversal en otras categorías a lo largo de este trabajo, por ejemplo: en que la mayoría de alternativas que encuentran a la sujeción mecánica sean equivalentes coercitivos, la presión grupal sentida para realizar sujeción mecánica, la percepción de la sujeción mecánica como algo imprescindible, etc. Con relación al modelo biomédico, alegan que la sujeción mecánica se indica en función de las etiquetas diagnósticas y/o a la conciencia de enfermedad y que influye en el malestar o el conflicto con la decisión tomada. 


\section{Conductas de los usuarios (justificación consciente del uso de sujeciones)}

Aunque reconocen la influencia que los aspectos anteriores tienen en la decisión de utilizar la sujeción mecánica, justifican que su uso tiene lugar en situaciones que se activan por determinadas acciones de los usuarios, cuya variabilidad entra en tensión con las presiones del contexto y sus creencias y valores. Las razones en que más frecuentemente basan su indicación son: 1) la interferencia en el funcionamiento institucional y la oposición al tratamiento pautado, 2) la prevención de riesgos, 3) evitar una fuga, y 4) el uso defensivo. En su experiencia, es habitual indicar una sujeción mecánica para forzar medidas que se consideran terapéuticas y necesarias (ingresos hospitalarios, tratamientos farmacológicos, observación en urgencias, reposo postprandial, etc.) a pesar de la negativa expresa de los usuarios y/o precisamente por ello, aunque también se ejecutan en ocasiones en las que la persona interfiere en el funcionamiento institucional, sin existir una clara oposición al tratamiento ni un riesgo inminente. La prevención de riesgos (específicos o indeterminados) es otra de las justificaciones en que sostienen el uso de sujeción mecánica, que funcionaría como medida de seguridad frente algo que el profesional teme que ocurra. El punto 3 (evitar una fuga), hace referencia a aquellas que se indican para evitar que los usuarios abandonen el hospital. Aunque en ella existen elementos tanto del punto 1 (oposición al tratamiento en contra del criterio médico), como del punto 2 (prevenir un posible riesgo), se plantea de modo independiente por su frecuencia como algo específico en su discurso. El uso defensivo de las sujeciones reúne las acciones de los usuarios (oponerse al tratamiento, intentar abandonar el hospital en contra del criterio médico, posibilidad de alteraciones de conducta, etc.), que activan en el profesional la indicación de sujeción mecánica por miedo a las posibles consecuencias legales o institucionales si no se efectúa. Por último, los residentes perciben que en ocasiones la sujeción mecánica se utiliza como una medida disciplinaria, es decir, como medida de castigo, para corregir una conducta, marcar la autoridad, etc., lo que es significativamente alarmante.

\section{Experiencias durante la indicación de sujeciones mecánicas}

En su mayoría, refieren que existen dos tipos de "presiones" que los empujan a tomar la decisión de indicar sujeción mecánica, y que aparecen como consecuencia de la interacción de los puntos anteriores: los factores ambientales y contextuales, las conductas de las personas usuarias y su propio sistema de creencias previo. Estas pueden experimentarse como miedos a las consecuencias de no efectuar la sujeción, y/o como presiones externas para que se realicen. Con frecuencia, describen que la indicación que se realiza puede ser inadecuada. Los factores que pueden modificar la decisión hacia una dirección diferente o bien reforzar la indicación de sujeción mecánica, se desarrollan más adelante.

\section{Percepción de ser presionado a sujetar: atrapado en demandas contradictorias}

Los residentes describen la sensación de encontrarse atrapados en demandas contradictorias difíciles de resolver: responsabilidad legal versus voluntad del usuario, o mandatos institucionales que, en sí, son contradictorios. Asimismo, señalan sentirse presionados a realizar sujeciones mecánicas por parte de diferentes agentes: otros residentes, facultativos, compañeros de otras profesiones, la institución, por su deber profesional de control social, etc. Esta presión se puede resolver cuando indican la sujeción mecánica, mientras que si no se realiza persiste como fuente de conflicto o tensión.

\section{Miedo}

Es uno de los puntos más relevantes. El miedo ocupa un lugar primordial en la experiencia emocional en relación con la indicación de 
las sujeciones. Se exponen los resultados ordenados de mayor a menor frecuencia de aparición según el objeto con que relacionan este miedo, aunque en muchas ocasiones puede encadenar distintas situaciones temidas, por ejemplo: miedo a que el usuario se fugue del hospital, se autolesione, esto tenga unas repercusiones institucionales y, además, una posible responsabilidad legal.

1. A que "pueda pasar algo": es la experiencia de un miedo a que algo, inespecífico, pero no deseado, pudiera pasar. Se asemeja más bien a una preocupación porque ocurra algo considerado perjudicial, fuera del control profesional, aunque bajo su responsabilidad, y que, por tanto, les hace sentir empujados a tomar una decisión para evitarlo.

2. A la autoagresión: a que el usuario se haga daño a sí mismo.

3. A las consecuencias legales: agrupa aqueIlas experiencias que describen el temor de posibles consecuencias legales como motor que empuja a indicar sujeción mecánica. Describen, también, un sentimiento de especial desprotección de los psiquiatras en relación con la toma de este tipo de decisiones.

4. A ser agredido: también es común el miedo a sufrir una agresión. La angustia por estar en peligro tiene base en experiencias personales previas o en experiencias colectivas que se han compartido. Se considera una indicación "clara" de "contención".

5. A la respuesta institucional: describen la experiencia de usar la sujeción mecánica con base en el miedo a la respuesta institucional, del propio servicio o de su adjunto (amonestación, reprimenda, queja, etc.).

\section{Uso inadecuado de sujeción mecánica}

Se recoge que la sujeción mecánica se debería utilizar como último recurso, en determinadas situaciones de riesgo y tras haber agotado todas las alternativas. Sin embargo, los residentes señalan lo habitual de la inadecuada utilización e indicación de la sujeción mecánica más allá de lo esperado, según los protocolos: uso fuera de las indicaciones esperadas en el objetivo y la forma, espacios y tiempos inadecuados de uso, mala preparación, procedimiento, participación y coordinación, sobreutilización, falta de cuidados en la Urgencia, etc. El mal uso de la sujeción mecánica fue una de las categorías principales referidas, y apoya la idea de que, en la situación actual, existe un riesgo importante de mal uso y abuso.

\section{Indicaciones alternativas a la sujeción mecánica}

Los profesionales identifican prácticas y estrategias que funcionan como sustitutos o alternativas a la sujeción mecánica. Un grupo de ellas, no suponen realmente una alternativa a la práctica de medidas coercitivas si no otras formas de coacción o equivalentes coercitivos. Entre ellos, encontramos: 1) amenazar con el uso de una sujeción mecánica; 2) dejar las sujeciones preparadas en la cama; 3 ) ingresar en unidades cerradas; 4) presencia de personal de seguridad; 5) contenciones físicas; 6) aislamiento; 7) traslados forzosos; y 8) coerción informal. No obstante, también identifican prácticas y medidas que consideran que permitirían reducir o evitar el uso de sujeciones sin que sean elementos coercitivos: individualizar las estrategias para afrontar las crisis, contrato terapéutico y voluntades anticipadas, acompañamiento por una persona querida, contención verbal, ejercicios basados en la atención o regulación emocional, habitaciones acolchadas, unidades de puertas abiertas, y atención y hospitalización en el domicilio. Algunas de estas alternativas son estructurales, otras tienen que ver con la anticipación a los momentos de crisis y otras con el abordaje en la propia crisis.

\section{Experiencias tras el uso de la sujeción mecánica}

Dentro de las experiencias derivadas del uso de sujeciones encontramos varias categorías especialmente remarcables. 


\section{Impacto emocional}

A pesar de las dificultades que se encontraron para profundizar el mundo emocional de los participantes, el análisis del discurso desvela que el uso de las sujeciones produce en la gran mayoría un impacto emocional negativo, describiendo frecuentemente su uso como desagradable, duro o incómodo. Usar sujeciones genera culpa y remordimiento, impotencia y frustración, incluso rabia, cuando buscan y no encuentran una alternativa. Las sujeciones pueden dejar marcada la experiencia de los profesionales. Consideran, incluso, que algunas experiencias pueden ser traumáticas para ellos.

\section{Alivio de la presión y el miedo}

Con mucha menor frecuencia, refieren sentimientos de alivio, seguridad y tranquilidad por la disponibilidad y el empleo de sujeciones mecánicas. Estos sentimientos no aparecían de forma tan explícita ni tan en primer plano como otros. Sin embargo, pensamos que son un elemento fundamental en la perpetuación de la indicación de sujeciones: su uso resuelve la tensión generada por las presiones del contexto y los miedos activados por las conductas de los usuarios, disminuyendo la preocupación por los riesgos y repercusiones legales o institucionales.

\section{Experiencias en conflicto}

El uso de la sujeción mecánica coloca a los residentes ante experiencias contradictorias o en conflicto. Según el análisis de los datos, podrían dividirse en tres, aunque están relacionadas:

1. Disonancia cognitiva: utilizamos este código para describir aquellas experiencias en las que sus creencias entran en contradicción entre ellas o, más habitualmente, cuando existe un conflicto entre las creencias y las emociones o conductas. Frecuentemente encontramos en su discurso que realizan sujeciones a pesar de mostrar ideas o deseos contrarios a ello. Entre otras cosas, relacionan la aplicación de sujeciones en contra de su propio criterio con la evitación del conflicto con el equipo, la jerarquía en la organización del trabajo o el miedo a la repercusión institucional.

2. Dilema ético: describe conflictos entre principios como beneficencia versus autonomía, o libertad versus seguridad. Señalan que existen tensiones entre lo que consideran que es su deber profesional y cómo se sienten con la práctica de la sujeción mecánica.

3. Conflicto con el rol profesional: describe el cuestionamiento como figura de cuidado que genera el uso de las sujeciones, que los coloca en conflicto con su rol profesional. Aparecen dos roles enfrentados: la figura que cuida y la que vigila/castiga. La escapatoria final a este conflicto es la incorporación de la creencia de que las sujeciones son parte indeseable, pero inevitable, de su trabajo y que no es posible negarse a realizarlas.

\section{Procesos de adaptación a la experiencia conflictiva}

Si la sujeción mecánica genera malestar emocional y experiencias disonantes, también a nivel moral, y experimentan que no hay posibilidad de negarse (en el contexto de la seguridad, de la jerarquía, del miedo a las consecuencias) resulta comprensible que, para poder trabajar en esta situación contradictoria y dañina, tengan lugar distintos procesos de adaptación. Los participantes describen cambios con el tiempo en su manera de sentir (les genera menos malestar), pensar (reflexionan menos acerca de ellas) y actuar, que definen como una habituación a la sujeción mecánica, una desensibilización en las reacciones y una automatización en su indicación.

\section{Estrategias y mecanismos para aliviar el malestar que producen las sujeciones}

Los profesionales reconocen el desarrollo de mecanismos no voluntarios (no conscientes), y de estrategias y acciones conscientes para disminuir el malestar que genera aplicar 
sujeción mecánica en su trabajo. Entre ellos, describen mecanismos de distracción, de distanciamiento de la experiencia emocional (intelectualización-negación-normalización) o de ventilación/expresión emocional.

\section{DISCUSIÓN}

\section{Cultura de la coerción, modelo biomédico y entornos incapacitantes}

Uno de los aspectos más significativos que se desprende de esta investigación es la existencia de una cultura institucional de la coerción que tiene, en su base, el modelo biomédico y la gestión del riesgo individual y social. Nuestros resultados respaldan lo señalado por Dainius Puras, Relator Especial de Naciones Unidas, en su informe sobre el derecho de toda persona al disfrute del más alto nivel posible de salud física y mental(22) sobre las bases que sustentan la justificación de las medidas coercitivas: "la necesidad médica y la peligrosidad". Los hallazgos apoyan que este enfoque tiene tal relevancia que impregna toda la organización institucional y las experiencias subjetivas de los profesionales ${ }^{(52)}$, a la vez que se encuentra en conexión con los marcos normativos y contextuales donde desarrollan su práctica, dando lugar a un modelo de trabajo basado en el paternalismo y la beneficencia, en la protección ${ }^{(79)}$ y evitación del riesgo ${ }^{(80)}$.

Nuestra investigación refleja la integración del modelo biomédico positivista en los profesionales a través del concepto de "falta de conciencia de enfermedad" y la influencia de las etiquetas diagnósticas en la interpretación de las conductas y discursos de los usuarios y las acciones que realizan. Aunque existen autores y organismos que han defendido la necesidad de expandir el modelo biomédico como medio para reducir el estigma ${ }^{(81,82,83)}$, nuestros resultados apoyan que este enfoque, que explica el sufrimiento mental a partir de teorías neurobiológicas, reduce la comprensión de las emociones y comportamientos de las personas diagnosticadas, no tiene (suficientemente) en cuenta los contextos y las relaciones ${ }^{(22)}$, intensifica la estigmatización ${ }^{(84)}$, favorece las actitudes paternalistas, y desprovee a las personas con sufrimiento mental de sus derechos, justificando la lesión de su autonomía con el objeto de alcanzar un bien superior ${ }^{(6,79)}$. Además, promueve una formación con pocas herramientas que faciliten la vinculación efectiva con los usuarios ${ }^{(80)}$. En nuestro estudio, se refleja en la tendencia a justificar el uso de sujeción mecánica basándose en la necesidad de intervenir sobre conductas (o posibles conductas) de las personas que son atendidas en salud mental, cuando se considera que precisan un tratamiento indispensable, incluso independientemente de que la persona haya realizado o no conductas "peligrosas", si se considera que no dispone de una capacidad adecuada para comprender qué es lo mejor para ella. Este concepto, interiorizado, de la falta de conciencia de enfermedad, sirve de apoyo epistemológico a los profesionales para limitar o suspender la autonomía del sujeto, y facilita el desplazamiento de la atención desde los significados y valores de la persona que sufre, hacia las conductas y su evaluación, ya que no se considera su discurso más que desde la enfermedad ${ }^{(6)}$.

En este sentido, nuestra investigación hace evidente la prioridad que tiene la evaluación y gestión del riesgo o peligrosidad en la práctica habitual. Por encima de las necesidades del individuo, la gestión del riesgo es un principio esencial en salud mental ${ }^{(18)}$. Otros estudios también han sugerido ${ }^{(28)}$ que la coerción sea una de las primeras respuestas que aparecen en el personal cuando perciben que su seguridad o la de otros ha estado en riesgo ${ }^{(85,86)}$, pero también que existe una sobrestimación del riesgo basado en la valoración de la conducta del usuario(28,86), y que esta sobreestimación de la amenaza percibida por los profesionales se relaciona con el miedo a incidentes violentos previos, lo cual impide que se piense en alternativas de cuidado no coercitivo ${ }^{(28,85)}$. De hecho, la consideración de las personas diagnosticadas con trastornos mentales como peligrosas no se corresponde con la evidencia ${ }^{(87)}$ y tiende a fundamentarse en prejuicios inapropiados ${ }^{(22)}$. 
Por otro lado, resulta paradójica la consideración de la sujeción mecánica como medida de seguridad cuando los propios profesionales admiten el impacto perjudicial que tienen sobre ellos, los usuarios y el vínculo terapéutico, respaldando los hallazgos de otros autores ${ }^{(25,33,88,89,90)}$. Por este y otros motivos, se ha considerado necesario transitar del modelo en el que la sujeción mecánica es considerada una herramienta que provee seguridad, a otro donde se la reconoce como acontecimiento centinela, como un evento indeseable, inesperado, que se acompaña de riesgos y/o lesiones, algunas de ellas de gravedad y que, por tanto, requiere de la implantación de medidas para reducirla-eliminarla ${ }^{(56)}$.

Esta conceptualización tiene consecuencias en la clínica, ya que la mayoría de alternativas que se plantean tienen que ver con pensar en otras formas de control conductual y de evitar riesgos, más que con la transformación del paradigma que los genera y los sustenta $^{(35)}$. Nuestro análisis sugiere que la coerción funciona en un espectro continuo, de modo que sufrir una medida coercitiva o haberla sufrido en el pasado, predispone a nuevas medidas coercitivas ${ }^{(3,6)}$.

En consonancia con sus discursos, la atribución de peligrosidad e incapacidad a las personas con sufrimiento emocional se encuentra en diálogo con un conjunto de sistemas legislativos-normativos que no solo legitiman, sino que presionan y obligan a los profesionales para que utilicen medidas coercitivas, incluso de un modo preventivo, como un mandato social(6), culpabilizando a estos profesionales si aparecen conductas violentas ${ }^{(80)}$ que, por otro lado, son siempre difícilmente predecibles ${ }^{(91)}$. Existen autores que mencionan el sentimiento de los profesionales de ser observados y evaluados ${ }^{(39)}$. De hecho, los discursos de los residentes sobre la sujeción mecánica no pueden separarse de términos relacionados con la norma, el deber y la seguridad. En una revisión reciente ${ }^{(92)}$, se ilustra una particularidad del marco jurídico español que lo diferencia de otros países, ya que el precepto legal que regula el internamiento involuntario ${ }^{(5)}$ no menciona en ningún momento la involuntariedad del tratamiento, haciendo recaer directamente sobre los profesionales la responsabilidad de aplicar, desde ese momento, cualquier otra medida que consideren necesaria, incluyendo la sujeción mecánica.

La generalizada infradotación de recursos a los sistemas de salud mental que evidencia el Relator ${ }^{(22)}$ parece acorde a la experiencia de los participantes, que denuncian las carencias materiales, estructurales y la inadecuación de los lugares de trabajo que, en vez de promover el bienestar, son espacios incapacitantes que dificultan el empleo de alternativas no coercitivas $^{(88,93)}$. Algunos investigadores no consideran que existan pruebas definitivas que justifiquen que las diferencias en la incidencia de medidas coercitivas se deban a carencias en la dotación del personal, la financiación de los servicios o la ratio profesionales-usuarios, y sugieren que estas se basan esencialmente en los factores culturales, las políticas y las tradiciones de cada lugar ${ }^{(17)}$. Sin embargo, otros consideran que para no utilizar sujeción mecánica los recursos son fundamentales, incluido el tiempo que se puede dedicar a los usuarios $^{(19)}$. De hecho, el déficit estructural y material derivado de la infrafinanciación se ha postulado como uno de los elementos que favorecen las violaciones de derechos humanos en psiquiatría ${ }^{(22)}$.

Los residentes identifican dinámicas en los equipos que favorecen el uso de las sujeciones. La jerarquía, en forma simbólica o bajo órdenes directas, es considerada como un factor que determina el uso de sujeciones, que se indican a pesar de sentir malestar o no compartir la decisión. Como señala Pértega, ciertas órdenes médicas se experimentan como difícilmente cuestionables a pesar de ser otro (residentes, enfermería etc.) quien las ejecuta ${ }^{(19)}$. La contribución de la estructura jerárquica en esta dinámica es fundamental, ya que genera una asimetría en la distribución de recursos asignados, la responsabilidad y separación de roles, espacios y tareas. Los residentes serían parte de aquellos no facultativos que, aunque pasan mucho más tiempo con el usuario, no tienen los mismos recursos ni capacidades para resolver determinadas situaciones, recurriendo al poder y la fuerza. 


\section{Riesgo de abusos y mal uso de las sujeciones mecánicas}

El relato de los residentes sobre la vivencia de una tendencia hacia el uso inadecuado de la sujeción mecánica, contrasta con otros estudios en los que los profesionales se muestran mayoritariamente de acuerdo en que el uso que se hace de ellas es "correcto"(94), lo que en nuestra opinión incita con más énfasis a que se revise el uso de estas prácticas, sobre la que no hemos encontrado investigaciones en nuestra región con las que poder comparar los resultados. Por otro lado, de los resultados destacan algunos conceptos que pueden considerarse problemáticos: "agitación", "terapéutico", "peligrosidad", "conciencia de enfermedad", "riesgo de fuga", "último recurso", etc. ${ }^{(88,95)}$. Todos ellos son básicos para armar el discurso que fundamenta el uso de las sujeciones $y$, sin embargo, en la experiencia de los profesionales resultan inespecíficos, polisémicos y subjetivos. Están abiertos a una amplia interpretación, no son respaldados por la investigación y, según el Relator ${ }^{(22)}$, podrían favorecer la arbitrariedad de la utilización de la sujeción mecánica ${ }^{(21)}$. La percepción que tienen los residentes acerca de los criterios de las sujeciones es que, al igual que el diagnóstico, la valoración de los usuarios y de los riesgos tiene un importante componente subjetivo, y que los protocolos no sirven para guiar las acciones de los profesionales con relación a estas medidas, en conformidad con los hallazgos de otros trabajos ${ }^{(19)}$.

\section{Sistema de creencias, miedos, inseguridad e incertidumbre}

En paralelo con el concepto bourdiano de habitus, consideramos que existe una correspondencia entre estructuras mentales y sociales-institucionales ${ }^{(96)}$. De este modo, las percepciones y experiencias de los profesionales, las categorías de las representaciones y visiones que tienen del mundo, son producto de la incorporación de estructuras del espacio social. Es decir, más allá de los hechos objetivos, su experiencia depende de la interiorización de esquemas del mundo, que informan sus percepciones, sentimientos y acciones. A partir de la interacción del sujeto-profesional con la cultura de grupo y las instituciones, se construye un sistema de disposiciones que son a su vez estructurales y estructurantes ${ }^{(97)}$. Así, lo que hemos denominado cultura de la coerción, influye los sistemas simbólicos e informa las percepciones y acciones de los residentes quienes, a su vez, son agentes activos en la construcción de la realidad en el espacio clínico-institucional. Las relaciones de poder, las jerarquías, la conceptualización del sufrimiento desde el modelo biomédico y los otros elementos de esta cultura, se interiorizan e inclinan a los residentes a percibir la clínica actual como evidente y natural. La consideración de la sujeción mecánica como imprescindible, el miedo a su eliminación, la asimilación con el tiempo como algo que forma parte de su trabajo y la tendencia a justificar su utilización, podrían entenderse como producto de la interiorización de esta cultura que, al reproducirla, perpetúan. Estas experiencias han sido expuestas también por otros investigadores ${ }^{(98,99)}$.

Asimismo, parece existir una trasposición del sentimiento de inseguridad e imprevisibilidad de lo social a la red de salud mental. La existencia de experiencias y elementos similares han sido descritos por Pértega ${ }^{(19)}$ : el fracaso en el establecimiento de criterios objetivos que ayuden a los profesionales ante el mandato social (y legal) delegado de evaluar y gestionar los riesgos, sumado a la imposibilidad real de prevenir y controlar toda la inestabilidad de los entornos y conductas de los usuarios, sumerge a los residentes en una incertidumbre-inseguridad en la que la sujeción mecánica sirve para generar certezas en unos profesionales responsables de una tarea imposible. De hecho, se observa en varios de los participantes la preocupación hacia una posible eliminación de la sujeción mecánica, sin la cual se sienten desprotegidos.

En relación con el proceso psicológico que subyace al uso de la sujeción mecánica en esta situación, el miedo aparece como parte primordial de la experiencia profesional, 
tanto hacia los daños físicos reales, como a las consecuencias que pueden derivarse en caso de que se produzca el daño. En este clima de incertidumbre prima la evitación del riesgo sobre la posible merma de derechos y otros daños que puedan aparecer. Si bien se podría argumentar que el temor de los profesionales tiene una base sólida en experiencias previas en las que, por ejemplo, los profesionales han sido agredidos a causa de retrasar la indicación de una sujeción mecánica ${ }^{(100,101)}$, consideramos imprescindible que en el análisis de fenómenos tan complejos como la agresividad o la agitación psicomotriz, se tengan en cuenta otros factores (por ejemplo, relacionales) o se reflexione acerca de cómo la falta de preparación o de conciencia sobre la influencia que uno mismo y el entorno ejercen sobre las personas, puede determinar que exista o no una situación de estas características ${ }^{(93,102)}$. En esta línea, el concepto de "escenas temidas"(103) que utiliza Dozza para nombrar aquellas situaciones imaginadas que tememos que ocurran, y que se caracterizan por acompañarse de desconcierto, de no saber cómo responder, de una sensación de falta de habilidades y herramientas para resolver la situación imaginada ${ }^{(104)}$, podría explicar la influencia que las creencias colectivas ejercen en la toma de decisiones de los profesionales. De sus discursos puede entenderse que, en ocasiones, el uso de las sujeciones vaya más dirigido a evitar esa escena temida que hacia las necesidades y cuidados que el usuario precisa. Las escenas guardan una estrecha relación con "fantasías catastróficas", es decir, situaciones por las cuales un profesional podría ser condenado o penalizado por la institución (suicidio, asesinatos, peleas), o bien aquellas en las que podemos ser dañados nosotros mismos (física, psicológica o laboralmente, etc. ${ }^{(104)}$. Estas fantasías, que en general no son conscientes, producirían toda una serie de emociones y cogniciones que empujarían a los profesionales a adoptar conductas conservadoras o sobreprotectoras, en ocasiones estereotipadas, a fin de evitar riesgos. Muchas veces, las fantasías y escenas son compartidas de manera colectiva (institución, sociedad, equipos, etc.) y tienen una influencia significativa en los sujetos que inician su formación a través de la cultura o filosofía de trabajo ${ }^{(105)}$.

\section{Impacto emocional y procesos de adaptación}

El impacto negativo de las sujeción mecánica a nivel emocional o psicológico ha sido referido en múltiples estudios ${ }^{(25,106)}$. Culpa, remordimiento, impotencia, rabia o frustración son emociones referidas frecuentemente ${ }^{(52)}$. En este sentido, Bloom utiliza el concepto de procesos paralelos para referirse al efecto simétrico que el uso del poder para manejar las alteraciones conductuales tiene en profesionales y usuarios: por un lado, hace que los usuarios teman, desconfíen del staff y sean menos colaboradores y participativos pero, al mismo tiempo, provoca que los profesionales se sientan frustrados e insatisfechos, lo que favorece que recurran aún más al uso del control y el poder ${ }^{(107)}$. Pero, ¿cómo se pasa del impacto que sigue a su utilización, a la aceptación y reproducción de estas medidas? Dentro de los procesos psicológicos observados, los residentes describen el desarrollo de formas de adaptación similares a las descritas por Pértega ${ }^{(19)}$. Los participantes narran una transformación en el tiempo en la forma en que viven la sujeción mecánica. Según lo observado, progresivamente, y a través de distintos mecanismos psicológicos pasivos y activos, tiene lugar una desensibilización, habituación y automatización de su uso, con disminución del malestar asociado y la integración de estas medidas como propias de su práctica habitual, disminuyendo también el conflicto generado por los dilemas éticos y la disonancia entre creencias y acciones. El proceso identificado por Pértega podría explicar la adaptación los residentes a través de tres fases consecutivas durante su formación: 1) traumatización y extrañamiento en las primeras sujeciones mecánicas; 2) racionalización, frustración e impotencia como defensa frente a la angustia que generan las medidas; y 3) incorporación y aceptación de las sujeción mecánica, momento en el que 
pasan a considerarse como una "parte del trabajo" $^{\prime \prime(19,89)}$. Sin embargo, Pértega sugiere que aparece también la sensación de haberse vuelto más defensivo, menos seguro de uno mismo en el trabajo, la creencia de que uno debe de protegerse, sin dejar nunca de desaparecer una sensación de extrañeza y de cuestionamiento del trabajo de uno mismo.

\section{Experiencias en conflicto}

Por último, no todos los estudios existentes recogen experiencias de los profesionales de carácter conflictivo con las medidas coercitivas. Por ejemplo, medidas coercitivas como el aislamiento han sido descritas por algunos profesionales como "muy necesarias" y "altamente terapéuticas", y han sugerido que garantizan la seguridad sin resultar punitivas $^{(108)}$. Otros investigadores encuentran que la mayoría de profesionales ven las medidas coercitivas como necesarias para garantizar la atención y la seguridad, poniendo en duda que puedan aparecer conflictos morales significativos ${ }^{(109)}$. No obstante, los resultados de nuestro trabajo ilustran que la experiencia compartida por la mayoría de residentes de nuestro contexto se caracteriza por presentar múltiples contradicciones que se despiertan cuando deciden indicar o realizar una sujeción mecánica. Esta diferencia podría deberse a que la denuncia sobre las consecuencias de la sujeción mecánica y los dilemas que conlleva su uso se han hecho mucho más presentes en el discurso profesional y público en los últimos años. Tener en cuenta que la sujeción mecánica es una medida necesaria pero dañina para el usuario y el vínculo terapéutico, coloca a los residentes ante conflictos que, para resolverse, muchas veces caen en la autojustificación, la negación de la disonancia ${ }^{(99)}$ y la aceptación de esta contradicción inherente a la psiquiatría actual. Esto puede verse en el proceso de toma de decisiones ${ }^{(19)}$, en el conflicto con el rol profesional(89) $\mathrm{o}$ en los dilemas éticos que se producen ${ }^{(37)}$.

\section{Fortalezas y limitaciones}

Una de las principales fortalezas se debe al aporte novedoso que supone para la bibliografía sobre la sujeción mecánica en nuestro contexto, en la que es llamativa la escasez de trabajos al respecto. Además, contrasta con la mayoría de publicaciones anglosajonas, que suelen estar centradas, particularmente, en el personal de enfermería. Haber considerado las vivencias de los residentes de distintas especialidades, enriquece la comprensión del fenómeno y permitirá hacer comparaciones con profesionales de mayor experiencia. Entre las limitaciones, destaca que la controversia que despierta el debate de la sujeción mecánica lo convierte en un tema de elevada deseabilidad social, la cual pudo influir en los relatos de los participantes. Además, aunque se utilizaron herramientas metodológicas para favorecer la representatividad de la muestra y la heterogeneidad de los discursos, es posible que aquellos con mayor indiferencia hacia estas prácticas, o que se puedan sentir amenazados por la exposición, no se hayan prestado a participar. Por otro lado, los datos recogidos deben entenderse dentro de un contexto temporal y espacial determinado, por lo que sería importante replicar este estudio en otros ámbitos a fin de lograr una comprensión más profunda del fenómeno allí donde tiene lugar. Por último, con relación al análisis, debe reconocerse la influencia de la propia historia y subjetividad de los investigadores, quienes han estado en contacto con el uso de sujeción mecánica en su práctica profesional, aunque no tiene por qué entenderse tan solo como una limitación, ya que fue un factor que enriqueció el diseño y desarrollo de estudio.

\section{CONCLUSIÓN}

El propósito de este trabajo ha sido explorar en profundidad la experiencia de los residentes de salud mental con relación al uso de sujeción mecánica y conocer qué aspectos y de qué manera influyen en el proceso de decidir su aplicación. Nuestros resultados 
se asemejan a los presentados por otros autores $^{(19,52,88,93,99,110,111)}$ y sugieren que los procesos de decisión del uso de sujeciones mecánicas se encuentran dentro de un complejo entramado de factores y experiencias en las que existen elementos del contexto sociocultural y el marco normativo, de dinámicas relacionales y de los entornos de trabajo, de procesos vivenciales, psicológicos y éticos, así como otros que resultan de la interacción entre sí. Entre ellos, destaca una cultura de trabajo basada en la coerción y el discurso de la gestión del riesgo. Los profesionales, a pesar de reconocer el impacto perjudicial que se deriva de su uso, atraviesan un proceso de adaptación por el que interiorizan y actúan con base en estos principios, justificando la necesidad de la sujeción mecánica a la vez que enfrentan conflictos con su rol profesional y dilemas éticos.

\section{Implicaciones en la práctica clínica}

En todo caso, los resultados de esta investigación nos empujan a exigir un cambio radical de paradigma que promueva una metamorfosis de nuestra práctica. Esto implica que las transformaciones deberán trascender áreas concretas de intervención y perseguir cambios políticos, legislativos, institucionales y culturales, sin dejar de atender el papel de los equipos y los profesionales. El uso de sujeciones mecánicas, por sus implicaciones, debe reconocerse como un fracaso de los cuidados y una medida a extinguir. Nuestro estudio sugiere caminos para transitar por la complejidad que rodea a este fenómeno hacia su eliminación.

\section{CONFLICTOS DE INTERÉS}

Este proyecto no ha contado con ningún tipo de financiación. Investigadores y participantes estuvieron exentos de cualquier beneficio económico o compensación, de manera directa o indirecta. No se han identificado intereses particulares (económicos, académicos u otros) que pudiesen entrar en conflicto con el estricto interés científico de la investigación.

\section{AGRADECIMIENTOS}

A todos los residentes que participaron en la investigación. A Ana Moreno y Amalia Fariña por sus consejos, apoyo y comentarios.

\section{REFERENCIAS BIBLIOGRÁFICAS}

1. Huertas García-Alejo R. Historia cultural de la psiquiatría. Madrid: La Catarata; 2012.

2. Kallert TW, Mezzich JE, Monahan J. Coercive treatment in psychiatry: Clinical, legal and ethical aspects. West Sussex: John Wiley \& Sons; 2011.

3. Kirk SA, Gomory T, Cohen D. Mad Science: Psychiatric coercion, diagnosis, and drugs. New Brunswick: Transaction Publishers; 2013.
4. European Committee for the Prevention of Torture and Inhuman or Degrading Treatment or Punishment. Means of restraint in psychiatric establishments for adults (Revised CPT standards) [Internet]. Estrasburgo; 2017 [citado 10 mar 2020]. Disponible en: https://rm.coe. int/16807001c3.

5. España, Jefatura del Estado. Ley $1 / 2000$, de 7 de enero, de Enjuiciamiento Civil [Internet]. BOE-A-2000-323 [citado 10 mar 2020]. Disponible en: https://tinyurl.com/ w7dywu9f.

6. Inchauspe Aróstegui JA, Valverde Eizaguirre M. La coerción en Salud Mental: conceptos, procesos y situación. En: Beviá B, Bono Á, editores. Coerción y salud mental: revisando las prácticas de coerción en la atención a las personas que utilizan los servicios de salud mental. Madrid: Asociación Española de Neuropsiquiatría; 2017. p. 13-89.

7. Kallert TW, Mezzich JE, Monahan J. Introduction. En: Kallert TW, Mezzich JE, Monahan J, editors. Coercive treatment in psychiatry: Clinical, legal and ethical aspects. West Sussex: John Wiley \& Sons; 2011. p. xi-xxii.

8. Mayoral F, Torres F, Group Eunomia. Use of coercive measures in psychiatry. Actas Españolas de Psiquiatría. 2005;33(5):331-338. 
9. Kallert TW, Glöckner M, Onchev G, Raboch J, Karastergiou A, Solomon Z, et al. The EUNOMIA project on coercion in psychiatry: study design and preliminary data. World Psychiatry. 2005;4(3):168-172.

10. Simpson SA, Joesch JM, West II, Pasic J. Risk for physical restraint or seclusion in the psychiatric emergency service (PES). General Hospital Psychiatry. 2014;36(1):113-118. doi: http://dx.doi.org/10.1016/j. genhosppsych.2013.09.009.

11. Raguan B, Wolfovitz E, Gil E. Use of physical restraints in a general hospital: a Cross-sectional observational study. Israel Medical Association Journal. 2015;17:633638.

12. Krüger C, Mayer H, Haastert B, Meyer G. Use of physical restraints in acute hospitals in Germany: A multi-centre cross-sectional study. International Journal of Nursing Studies. 2013;50:1599-1606. doi: 10.1016/j. ijnurstu.2013.05.005.

13. Foebel AD, Onder G, Finne-soveri $H$, Lukas A, Denkinger MD, Carfi A, et al. Physical restraint and antipsychotic medication use among nursing home residents with dementia. Journal of the American Medical Directors Association. 2016;17(2):184.e9-184.e14.

14. Hui A, Middleton H, Völlm B. The uses of coercive measures in forensic psychiatry: A literature review. In: Völlm B, Nedopil N, editors. The use of coercive measures in forensic psychiatric care: Legal, ethical and practical challenges. Switzerland: Springer; 2016. p. 151-184

15. European Committee for the Prevention of Torture and Inhuman or Degrading Treatment or Punishment. CPT/Inf (2017) 34, Section: 24/41, Date: 06/04/2017, B Prison establishments, 5 Means of restraint [Internet]. 2017 [10 mar 2020]. Disponible en: https://tinyurl.com/5h53bdym.

16. Steinert T, Lepping P. Legal provisions and practice in the management of violent patients: A case vignette study in 16 European countries. European Psychiatry. 2009;24(2):135-141. doi: 10.1016/j.eurpsy.2008.03.002.

17. Steinert $T$, Lepping $P$, Bernhardsgrütter $R$, Conca A, Hatling $T$, Janssen $W$, et al. Incidence of seclusion and restraint in psychiatric hospitals: a literature review and survey of international trends. Social Psychiatry and Psychiatric Epidemiology. 2010;45(9):889-897. doi: 10.1007/s00127-009-0132-3

18. Sashidharan S, Mezzina R, Puras D. Reducing coercion in mental healthcare. Epidemiology and Psychiatric Sciences. 2019;28(6):605-612. doi: 10.1017/ S2045796019000350.

19. Pértega E. Health professionals' decision making process about the use of physical restraints in inpatient pediatric psychiatric units in Madrid. New York University, Universidad Autónoma de Madrid; 2015.

20. Organización de las Naciones Unidas. Convención sobre los derechos de las personas con discapacidad [Internet]. Nueva York; 2006 [citado 10 mar 2020]. Disponible en: https://tinyurl.com/49f6p5bn.
21. Naciones Unidas, Asamblea General. Informe del Relator Especial sobre la tortura y otros tratos o penas crueles, inhumanos o degradantes [Internet]. Naciones Unidas; 2016 [citado 10 mar 2020]. Disponible en: https://tinyurl.com/dvw9t34e.

22. Consejo de Derechos Humanos. Informe del Relator Especial sobre el derecho de toda persona al disfrute del más alto nivel posible de salud física y mental [Internet]. 2017 [citado 10 mar 2020]. Disponible en: https:// tinyurl.com/wcj85urf

23. Szmukler G. Compulsion and "coercion" in mental health care. World Psychiatry. 2015;14(3):259-261. doi: 10.1002/wps.20264.

24. Brodwin P. The ethics of ambivalence and the practice of constraint in US psychiatry. Culture, Medicine and Psychiatry. 2014;38(4):527-549. doi: 10.1007/s11013-014-9401-z.

25. Sweeney A, Filson B, Kennedy A, Collinson L, Gillard S. A paradigm shift: relationships in trauma-informed mental health services. BJPsych Advances. 2018;24(5): 319-333.

26. Sailas E, Fenton M. Seclusion and restraint for people with serious mental illnesses. Cochrane Database Syst Rev. 2000;(1):CD001163. doi: 10.1002/14651858. CD001163.

27. Luciano M, Sampogna G, Vecchio V Del, Pingani $\mathrm{L}$, Palumbo C, Rosa C De, et al. Use of coercive measures in mental health practice and its impact on outcome: a critical review. Expert Review of Neurotherapeutics. 2014;14(2):131-141. doi: 10.1586/14737175. 2014.874286

28. Cusack P, Cusack FP, McAndrew S, McKeown M, Duxbury J. An integrative review exploring the physical and psychological harm inherent in using restraint in mental health inpatient settings. International Journal of Mental Health Nursirg. 2018;27(3):1162-1176.

29. Cusack P, Mcandrew S, Cusack F, Warne T. Reviewing evidence of the effects of restraint from the perspective of service users and mental health professionals in the United Kingdom (UK). International Journal of Law and Psychiatry. 2016;46:20-26. http://dx.doi. org/10.1016/j.ijlp.2016.02.023.

30. Rose D, Evans J, Laker C, Wykes T. Life in acute mental health settings: experiences and perceptions of service users and nurses. Epidemiology of Psychiatric Sciences. 2015;24(1):90-96.

31. Duxbury JA. The Eileen Skellern Lecture 2014: physical restraint : in defence of the indefensible? Journal of Psychiatric and Mental Health Nursing. 2015;22(2):92101. https://doi.org/10.1111/jpm.12204.

32. Rakhmatullina M, Taub A, Jacob T. Morbidity and mortality associated with the utilization of restraints: A review of literature. The Psychiatric Quarterly. 2013;84(4):499-512. doi: 10.1007/s11126-013-9262-6. 
33. Muskett $C$. Trauma-informed care in inpatient mental health settings : A review of the literature. International Journal of Mental Health Nursing. 2014;23(1):51-59. doi: 10.1111/inm.12012.

34. Colectivo Locomún. \#0 Contenciones [Internet]. 2018 [citado 25 sep 2019]. Disponible en: https://tinyurl.com/ hmwpnzx6.

35. Gooding P, Mcsherry B, Roper C, Grey F. Alternatives to coercion in mental health settings: A literature review [Internet]. Melbourne: Melbourne Social Equity Institute, University of Melbourne; 2018 [citado $10 \mathrm{sep}$ 2019]. Disponible en: https://tinyurl.com/3y7jf98y.

36. Comisión de Sanidad y Servicios Sociales. Proposición no de Ley presentada por el Grupo Parlamentario Ciudadanos, relativa a la eliminación de las sujeciones mecánicas en el ámbito asistencial: Aprobación con modificaciones así como enmiendas formuladas. Boletín Oficial de las Cortes Generales Madrid [Internet]. 2017 [citado 10 sep 2019]. Disponible en: https://tinyurl.com/ pw4kvsww.

37. Hem MH, Gjerberg E, Husum TL, Pedersen R. Ethical challenges when using coercion in mental healthcare: A systematic literature review. Nursing Ethics. 2016;25(1):92-110. doi: 10.1177/0969733016629770.

38. Winship G. Further thoughts on the process of restraint. Psychiatric and Mental Health Nursing. 2006;13:55-60. doi: 10.1111/j.1365-2850.2006.00913.x

39. Beviá B, Girón M. Poder, estigma y coerción: Escenarios para una práctica no autoritaria en salud mental. Revista de la Asociación Española de Neuropsiquiatría. 2017;37(132):321-329. doi: 10.4321/s021157352017000200001

40. Canvin K, Rugkåsa J, Sinclair J, Burns T. Leverage and other informal pressures in community psychiatry in England. International Journal of Law and Psychiatry. 2013;36(2):100-106. doi: 10.1016/j.ijlp.2013.01.002

41. Raboch J, Kališová L, Nawka A, Kitzlerová E, Onchev G, Karastergiou A, et al. Use of Coercive Measures During Involuntary Hospitalization: Findings From Ten European Countries. Psychiatr Services. 2010;61(10):10121017. doi: 10.1176/ps.2010.61.10.1012

42. Duxbury J, Whittington R. Causes and management of patient aggression and violence: staff and patient perspectives. Journal of Advanced Nursing. 2005;50(5):469478. doi: 10.1111/j.1365-2648.2005.03426.x

43. Petti TA, Mohr WK, Somers JW, Sims L. Perceptions of Seclusion and Restraint by Patients and Staff in an Intermediate-Term Care Facility. Journal of Child and Adolescent Psychiatric Nursing. 2001;14(3):115-127. doi: 10.1111/j.1744-6171.2001.tb00303.x

44. European Committee for the Prevention of Torture and Inhuman or Degrading Treatment or Punishment. Means of restraint in a psychiatric hospital. Estrasburgo: CTP; 2006.
45. Marangos-Frost S, Wells D. Psychiatric nurses' thoughts and feelings about restraint use: a decision dilemma. Journal of Advanced Nursing. 2000;31(2):362369. doi: 10.1046/j.1365-2648.2000.01290.x

46. El-Badri S, Mellsop G. Patient and staff perspectives on the use of seclusion. Australasian Psychiatry. 2008;16(4):248-252. doi: 10.1080/10398560802027302.

47. Brophy LM, Roper CE, Hamilton BE, Tellez JJ, McSherry BM. Consumers and Carer perspectives on poor practice and the use of seclusion and restraint in mental health settings: Results from Australian focus groups. International Journal of Mental Health System. 2016;10(6). doi: 10.1186/s13033-016-0038-x.

48. Gudde CB, Olsø TM, Whittington R, Vatne S. Service users' experiences and views of aggressive situations in mental health care: A systematic review and thematic synthesis of qualitative studies. Journal of Multidisciplinary Healthcare. 2015;8:449-462.

49. Cornaggia CM, Beghi M, Pavone F, Barale F. Aggression in psychiatry wards: A systematic review. Psychiatry Research. 2011;189(1):10-20. doi: 10.1016/j. psychres.2010.12.024.

50. Happell B, Harrow A. Nurses' attitudes to the use of seclusion: A review of the literature. International Journal of Mental Health Nursing. 2010;19(3):162-168. doi: 10.1111/j.1447-0349.2010.00669.x.

51. Van Der Merwe M, Muir-Cochrane E, Jones J, Tziggili M, Bowers L. Improving seclusion practice: Implications of a review of staff and patient views. Journal of Psychiatry Mental Health Nursing. 2013;20(3):203-215. doi: 10.1111/j.1365-2850.2012.01903.x.

52. Cocho Santalla C, Nocete Navarro L, López Álvarez I, Carballeira Carrera L, Fernández Liria A. Experiencia y actitudes de los profesionales de salud mental en torno a las prácticas coercitivas directas: revisión bibliográfica de estudios cualitativos. Revista de la Asociación Española de Neuropsiquiatría. 2018;38(134):419-449. doi: 10.4321/S0211-57352018000200005.

53. Anderson S. Coercion. The Stanford Encyclopedia of Philosophy (Winter 2017) [Internet]. Edward NZ; 2017. Disponible en: https://tinyurl.com/8nrydmau.

54. Lovell A, Smith D, Johnson P. A qualitative investigation into nurses' perceptions of factors influencing staff injuries sustained during physical interventions employed in response to service user violence within one secure learning disability service. Journal of Clinical Nursing. 2015;24(13-14):1926-1935. doi: 10.1111/jocn.12830.

55. Strout TD. Perspectives on the experience of being physically restrained: An integrative review of the qualitative literature. International Journal of Mental Health Nursing. 2010;19(6):416-427. doi: 10.1111/j.1447-0349. 2010.00694.x.

56. Moreno Pérez A, Fernández Liria A. La contención mecánica como acontecimiento centinela: propuesta para avanzar hacia su eliminación. Boletín la Asociación Madrileña de Salud Mental. 2020;46. 
57. Knowles SF, Hearne J, Smith I. Physical restraint and the therapeutic relationship. The Journal of Forensic Psychiatry \& Psychology. 2015;26(4):461-475. doi: 10.1080/14789949.2015.1034752.

58. MohrWK, Petti TA, MohrBD. Adverse effects associated with physical restraint. Canadian journal of psychiatry. 2003;48:330-337. doi: 10.1177/070674370304800509.

59. Real Academia Española. Experiencia [Internet]. Diccionario de la Lengua Española. 2017 [citado 6 feb 2017]. Disponible en: https://tinyurl.com/5zrrnzn4.

60. Nocete Navarro L, Carballeira Carrera L, López Álvarez I, Cocho Santalla C, Fernández Liria A. Percepciones y actitudes de los profesionales de salud mental en la contención mecánica. En: Coerción y salud mental: revisando las prácticas de coerción en la atención a las personas que utilizan los servicios de salud mental. Madrid: Asociación Española de Neuropsiquiatría; 2017. p. 109-168.

61. Berenguera A, Fernandez de Sanmamed MJ, Pons M, Pujol E, Rodríguez D, Saura S. Escuchar, observar y comprender: Recuperando la narrativa en las Ciencias de la Salud: Aportaciones de la investigación cualitativa [Internet]. Barcelona: Institut Universitari d'Investigació en Atenció Primària Jordi Gol (IDIAP J. Gol); 2014. Disponible en: www.idiapjgol.org.

62. Onocko Campos RT, Furtado JP. Narrativas: utilização na pesquisa qualitativa em saúde. Revista de Saúde Publica. 2008;42(6):1090-1096. doi: 10.1590/ S0034-89102008005000052.

63. Prieto Rodríguez MA, March Cerdá JC. Paso a paso en el diseño de un estudio mediante grupos focales. Atención Primaria. 2002;29(6):366-373.

64. Laza Vásquez C, Pulido Acuña GP, Castiblanco Montañez RA. La fenomenología para el estudio de la experiencia de la gestación de alto riesgo. Enfermería Global. 2012;11(4):295-305

65. Eatough V, Smith J. I was like a wild wild person: Understanding feelings of anger using interpretative phenomenological analysis. British Journal of Psychology. 2006;(97):483-498. doi: 10.1348/000712606X97831.

66. Denzin NK, Lincoln YS. The SAGE Handbook of Qualitative Research. Newbury Park: SAGE Publications; 2011.

67. Husum TL, Bjørngaard JH, Finset A, Ruud T. Staff attitudes and thoughts about the use of coercion in acute psychiatric wards. Social Psychiatry and Psychiatric Epidemioly. 2011;46(9):893-901. doi: 10.1007/s00127010-0259-2.

68. Wynn R, Kvalvik AM, Hynnekleiv T. Attitudes to coercion at two Norwegian psychiatric units. Nordic Journal of Psychiatry. 2011;65(2):133-137. doi: 10.3109/ 08039488.2010.513068.

69. Thornberg R, Charmaz K. Grounded Theory and Theoretical Coding. En: Flick U, (ed). The SAGE Handbook of Qualitative Data Analysis. London: SAGE Publications; 2014. p. 153-169.
70. Tweed A, Charmaz K. Grounded Theory Methods for Mental Health Practitioners. En: Harper D, Thompson AR (eds). Qualitative Research Methods in Mental Health and Psychotherapy: A Guide for Students and Practitioners. West Sussex: John Wiley \& Sons; 2012. p. 131-146.

71. Olofsson B, Gilje F, Jacobsson L, Norberg A. Nurses narratives about using coercion in psychiatric care. Journal of advanced nursing. 1998;28(1):45-53. doi: 10.1046/j.1365-2648.1998.00687.x.

72. Corbin J, Holt NL. Grounded Theory. En: Somekh B, Lewin C (eds). Research Methods in the Social Sciences. London: SAGE Publications; 2005. p. 49-55.

73. Thomas J, Harden A. Methods for the thematic synthesis of qualitative research in systematic reviews. BMC Medical Research Methodoly. 2008;8(45). doi: 10.1186/1471-2288-8-45.

74. Calderón C. Criterios de calidad en la investigación cualitativa en salud (ICS): apuntes para un debate necesario. Revista Española de Salud Pública. 2002;76(5):473482.

75. Strauss A, Corbin J. Bases de la investigación cualitativa: técnicas y procedimientos para desarrollar la teoría fundamentada. Medellín: Editorial Universidad de Antioquia; 2002.

76. Emanuel EJ, Wendler D, Grady C. What Makes Clinical Research Ethical? Journal of the American Medical Association. 2000;283(20):2701-2711. doi: 10.1001/ jama.283.20.2701.

77. BOE. Ley Orgánica 15/1999, de 13 de diciembre, de Protección de Datos de Carácter Oficial. Boletín Of del Estado [Internet]. 1999;(298):43088-43099. Disponible en: https://tinyurl.com/vc48n29m

78. Asociación Médica Mundial. Declaración de Helsinki de la Asociación Médica Mundial: Principios Éticos para las Investigaciones Médicas en Seres Humanos [Internet]. Asociación Médica Mundial Helsinki; 1964. Disponible en: https://tinyurl.com/5v6ypvd6.

79. Szmukler G, Appelbaum PS. Treatment pressures, leverage, coercion, and compulsion in mental health care. Journal of the American Medical Association. 2008;17(3):233-244. doi: 10.1080/09638230802052203.

80. Bentall R. Too much coercion in mental health services. The Guardian [Internet]. 2013. Disponible en: https://tinyurl.com/2588jjz9.

81. Martin JK, Pescosolido BA, Tuch SA. Of fear and loathing: The role of "disturbing behavior," labels, and causal attributions in shaping public attitudes toward people with mental illness. Journal of Health and Socia Behavior. 2000;41(2):208-223. doi: 10.2307/2676306.

82. Malla A, Joober R, Garcia A. Mental illness is like any other medical illness: A critical examination of the statement and its impact on patient care and society. Journal of Psychiatry \& Neuroscience. 2015;40(3):147150. doi: 10.1503/jpn.150099. 
83. Angermeyer medidas coercitivas, Holzinger A, Carta MG, Schomerus G. Biogenetic explanations and public acceptance of mental illness: Systematic review of population studies. The British Journal of Psychiatry: the Journal of Mental Science. 2011;199(5):367-372. doi: 10.1192/bjp.bp.110.085563.

84. Read J, Haslam N, Magliano L. Prejudice, stigma and "schizophrenia": the role of the bio-genetic ideology." En: Read J, Dillon J (eds). Models of Madness: Psychological, Social and Biological approaches to psychosis. 2nd ed. East Sussex: Routledge; 2013.

85. Perkins E, Prosser H, Riley D, Whittington R. Physical restraint in a therapeutic setting; a necessary evil? The International Journal of Law and Psychiatry. 2012;35:43-49. doi: 10.1016/j.ijlp.2011.11.008.

86. Foster C, Bowers L, Nijman H. Aggressive behaviour on acute psychiatric wards. prevalence, severity and management. Journal of Advanced Nursing. 2007;58(2):140149. doi: 10.1111/j.1365-2648.2007.04169.x.

87. Peterson JK, Skeem J, Kennealy P, Bray B, Zvonkovic A. How often and how consistently do symptoms directly precede criminal behavior among offenders with mental illness? Law and Human Behavior. 2014;38(5):439-449. doi: $10.1037 / / h b 0000075$.

88. Riahi S, Thomson G, Duxbury J. An integrative review exploring decision-making factors influencing mental health nurses in the use of restraint. Journal of Psychiatric and Mental Health Nursing. 2016;23:116128. doi: 10.1111/jpm.12285.

89. Bigwood S, Crowe M. 'It's part of the job, but it spoils the job': A phenomenological study of physical restraint: Feature Article. International Journal of Mental Health Nursing. 2008;17(3):215-222. doi: 10.1111/j.14470349.2008.00526.x.

90. Sequeira $H$, Halstead S. The psychological effects on nursing staff of administering physical restraint in a secure psychiatric hohspital: When I go home, it's then that I think about it. British Journal of Forensic Practice. 2004;6(1):3. doi: 10.1108/14636646200400002.

91. Bowers L. On conflict, containment and the relationship between them. Nursing Inquiry. 2006;13(3):172180. doi: 10.1111/j.1440-1800.2006.00319.x.

92. Saya A, Brugnoli C, Piazzi G, Liberato D, Ciaccia G $\mathrm{Di}$, Niolu C, et al. Criteria, procedures, and future prospects of involuntary treatment in psychiatry around the world: A narrative review. Front Psychiatry. 2019;10. doi: 10.3389/fpsyt.2019.00271.

93. Bowers L. Safewards: A new model of conflict and containment on psychiatric wards. Journal of Psychiatric and Mental Health Nursing. 2014;21(6):499-508. doi: 10.1111/jpm.12129.

94. Wynn R. Staff's attitudes to the use of restraint and seclusion in a Norwegian university psychiatric hospital. Nordic Journal of Psychiatry. 2003;57(6):453-459. doi: 10.1080/08039480310003470.
95. Laiho $\mathrm{T}$, Kattainen E, Åstedt-Kurki P, Putkonen $\mathrm{H}$, Lindberg N, Kylmä J. Clinical decision making involved in secluding and restraining an adult psychiatric patient: An integrative literature review. Journal of Psychiatric and Mental Health Nursing. 2013;20(9):830-839. doi: 10.1111/jpm.12033.

96. Bourdieu P. La distinción: Criterio y bases sociales del gusto. Madrid: Gruop Santillana de Ediciones; 1998.

97. Capdevielle J. El concepto de habitus: “con Bordieu y contra Bordieu." Anduli: Revista Andaluza de Ciencias Sociales. 2011;(10):31-46.

98. Fisher WA. Restraint and Seclusion: A Review of the Literature. The American Journal of Psychiatry. 1994;151:1584-1591. doi: 10.1176/ajp.151.11.1584.

99. Jacob JD, Holmes D, Corneau P, Macphee C. Convergence and divergence: An analysis of mechanical restraints. Nursing Ethics. 2019;26(4):1009-1026. doi: 10.1177/0969733017736923.

100. Moylan LB, Cullinan M. Frequency of assault and severity of injury of psychiatric nurses in relation to the nurses' decision to restrain. Journal of psychiatric and mental health nursing. 2011;18(6):526-534. doi: 10.1111/j.1365-2850.2011.01699.x.

101. Khadivi AN, Patel RC, Atkinson AR, Levine JM. Association between seclusion and restraint and patientrelated violence. Psychiatric Services. 2004;55(11):13111312. doi: 10.1176/appi.ps.55.11.1311.

102. Moore G, Pfaff JA. Assessment and emergency management of the acutely agitated or violent adult. En: Waltham MA; Robert S Hockberger (ed). Uptodate; 2020.

103. Kesselman H, Pavlovsky E, Frydlevsky L. Las escenas temidas del coordinador de grupos. Clínica y Análisis Grupal. 1976;1(1):1-9.

104. Dozza de Mendoça L. Escenas temidas en salud mental [Internet]. Madrid; 2016 [citado 10 dic 2019]. Disponible en: https://tinyurl.com/y9whfp44

105. Vedana KGG, da Silva DM, Ventura CAA, Giacon BCC, Zanetti ACG, Miasso AI, et al. Physical and mechanical restraint in psychiatric units: Perceptions and experiences of nursing staff. Archives of Psychiatric Nursing. 2018;32(3):367-372. doi: 10.1016/j.apnu.2017.11.027.

106. Knight C. Trauma-Informed Social Work Practice: Practice Considerations and Challenges. Clinical Social Work Journal. 2015;43(1):25-37. doi: 10.1007/s10615014-0481-6.

107. Bloom SL. Human service systems and organizational stress: Thinking \& feeling our way out of existing organizational dilemmas [Internet]. Community Works; 2006 [citado 10 dic 2019]. Disponible en: https://tinyurl. com/w6ymrxs.

108. Meehan T, Bergen H, Fjeldsoe K. Staff and patient perceptions of seclusion: Has anything changed? Journal of Advanced Nursing. 2004;47(1):33-38. doi: 10.1111/j.1365-2648.2004.03062.x. 
109. Molewijk B, Kok A, Husum T, Pedersen R, Aasland O. Staff's normative attitudes towards coercion: The role of moral doubt and professional context: A cross-sectional survey study. BMC Medical Ethics. 2017;18(1):1-14. doi: 10.1186/s12910-017-0190-0.

110. Krieger E, Moritz S, Lincoln TM, Fischer R, Nagel M. Coercion in psychiatry: A cross-sectional study on staff views and emotions. Journal of Psychiatric and Mental Health Nursing. 2020;1-14. doi: 10.1111/jpm.12643.
111. Jacobsen TB. Involuntary treatment in Europe: different countries, different practices. Current opinion in psychiatry. 2012;25(4):307-310. doi: 10.1097/YCO.0b0 $13 \mathrm{e} 32835462 \mathrm{e} 3$.

\section{FORMA DE CITAR}

Nocete Navarro L, López de Loma Osorio V, Bravo Ortiz MF, Fernández Liria A. Salud mental y derechos humanos: La experiencia de los profesionales en formación en el uso de sujeciones mecánicas en Madrid, España. Salud Colectiva. 2021;17:e3045. doi: 10.18294/sc.2021.3045.

Recibido: 31 jul 2020 | Versión final: 15 dic 2020 | Aprobado: 28 dic 2020 | Publicado en línea: 23 mar 2021

Esta obra está bajo una licencia de Creative Commons Reconocimiento-NoComercial 4.0 Internacional. Reconocimiento - Permite copiar, distribuir y comunicar públicamente la obra. A cambio, se debe reconocer y citar al autor original. No Comercial - Esta obra no puede ser utilizada con finalidades comerciales, a menos que se obtenga el permiso. 